University of South Carolina

Scholar Commons

\title{
Globally Optimal Grouping for Symmetric Closed Boundaries by Combining Boundary and Region Information
}

Joachim S. Stahl

Song Wang

University of South Carolina - Columbia, songwang@cse.sc.edu

Follow this and additional works at: https://scholarcommons.sc.edu/csce_facpub

Part of the Computer Engineering Commons

\section{Publication Info}

Published in IEEE Transactions on Pattern Analysis and Machine Intelligence, Volume 30, Issue 3, 2008, pages 395-411.

http://ieeexplore.iee. $\mathrm{org} / \mathrm{xpl} /$ Recentlssue.jsp?punumber=34

(C) 2008 by the Institute of Electrical and Electronics Engineers (IEEE)

This Article is brought to you by the Computer Science and Engineering, Department of at Scholar Commons. It has been accepted for inclusion in Faculty Publications by an authorized administrator of Scholar Commons. For more information, please contact digres@mailbox.sc.edu. 


\title{
Globally Optimal Grouping for Symmetric Closed Boundaries by Combining Boundary and Region Information
}

\author{
Joachim S. Stahl, Student Member, IEEE, and Song Wang, Member, IEEE
}

\begin{abstract}
Many natural and man-made structures have a boundary that shows a certain level of bilateral symmetry, a property that plays an important role in both human and computer vision. In this paper, we present a new grouping method for detecting closed boundaries with symmetry. We first construct a new type of grouping token in the form of symmetric trapezoids by pairing line segments detected from the image. A closed boundary can then be achieved by connecting some trapezoids with a sequence of gap-filling quadrilaterals. For such a closed boundary, we define a unified grouping cost function in a ratio form: the numerator reflects the boundary information of proximity and symmetry, and the denominator reflects the region information of the enclosed area. The introduction of the region-area information in the denominator is able to avoid a bias toward shorter boundaries. We then develop a new graph model to represent the grouping tokens. In this new graph model, the grouping cost function can be encoded by carefully designed edge weights, and the desired optimal boundary corresponds to a special cycle with a minimum ratio-form cost. We finally show that such a cycle can be found in polynomial time using a previous graph algorithm. We implement this symmetry-grouping method and test it on a set of synthetic data and real images. The performance is compared to two previous grouping methods that do not consider symmetry in their grouping cost functions.
\end{abstract}

Index Terms-Perceptual organization, edge grouping, boundary detection, boundary symmetry, edge detection, graph models.

\section{INTRODUCTION}

$\mathrm{T}$ HE boundaries of many structures of interest encountered in the real world show a certain level of (bilateral) symmetry [4], [5]. For example, most objects (or their components) that are machine fabricated have a revolved surface that is perfectly symmetric over a straight axis. Many natural objects such as leaves and animals also have a boundary with a certain level of symmetry, where the symmetry axes may not be perfectly straight, as shown in Fig. 1c. In computer vision, symmetry has been shown to be an important property in both boundary interpretation/ matching, where the goal is to analyze and match given boundaries, and grouping, where the goal is to extract salient structural boundaries from real images [10]. As shown in Fig. 1, the goal of this paper is to develop an effective method to address the latter problem of grouping for symmetric boundaries, which, as pointed out in [10], is a particularly challenging problem.

Several reasons make the grouping for symmetric boundaries a challenging problem. First, unlike many other grouping cues, boundary symmetry is not a simple local measure: two symmetric fragments along the resulting boundary may be located far away from each other. As a result, it is usually difficult to encode symmetry into the simple locally constructed grouping tokens such as image pixels or boundary fragments, which have been widely

- The authors are with the Department of Computer Science and Engineering, University of South Carolina, Columbia, SC 29208.

E-mail: \{stahlj, songwang\}@engr.sc.edu.

Manuscript received 3 June 2006; revised 2 Feb. 2007; accepted 14 May 2007; published online 30 May 2007.

Recommended for acceptance by R. Basri.

For information on obtaining reprints of this article, please send e-mail to: tpami@computer.org, and reference IEEECS Log Number TPAMI-0416-0606. Digital Object Identifier no. 10.1109/TPAMI.2007.1186. used in previous grouping methods. Second, although symmetry is an important grouping cue, other cues, such as the Gestalt laws of proximity and closure are also crucial to achieve a successful grouping [16]. This calls for a unified grouping cost (function) that can flexibly integrate different grouping cues. Third, the grouping cost should be designed to avoid undesirable explicit or implicit biases such as a bias toward shorter boundaries, which occurs in many previous grouping methods [37]. Finally, it is usually a challenging problem to develop an optimization algorithm for finding a grouping that minimizes the selected grouping cost.

In this paper, we developed a new grouping method for detecting 2D closed boundaries with symmetry. Particularly, we propose a new grouping cost function that takes a ratio form: the numerator reflects the boundary information of proximity and symmetry, and the denominator reflects the region information of the enclosed area. The use of the enclosed region area makes the resulting grouping biased to detecting longer and rounder boundaries and therefore promotes the robustness to image noise and texture. This grouping cost function can be expanded to include other boundary information, such as boundary continuity (smoothness), and region information such as region intensity homogeneity. To quantify and encode this grouping cost, we construct a new type of grouping token in the form of trapezoids by pairing line segments detected from the image. Based on these trapezoids, the problem of grouping for symmetric boundaries can be formulated as identifying and connecting a sequence of trapezoids into a closed boundary. Finally, we construct a new graph model where candidate boundaries are represented as special cycles in this graph and apply a known graph algorithm to find the optimal cycle that corresponds to the boundary with the minimum grouping cost. Note that the work in this paper is only concerned with 


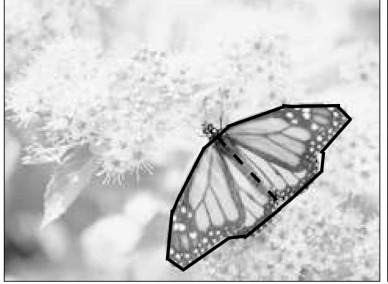

(a)

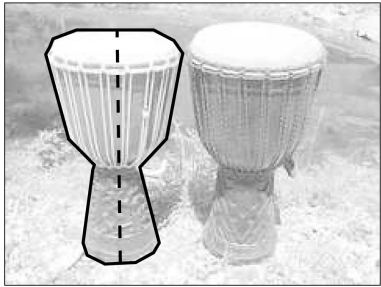

(b)

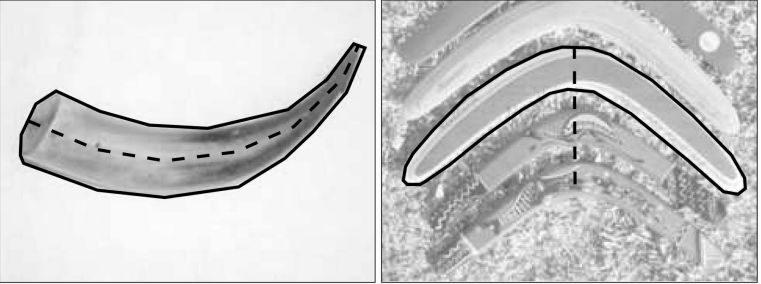

(c) (d)

Fig. 1. Four samples of structural boundaries that show symmetry. Structural boundaries are shown as solid curves, whereas the symmetry axes are shown as dashed curves.

the case where the boundary of interest shows bilateral symmetry on the 2D image plane. We do not consider the case where the surface of an object shows symmetry over an axis in $3 \mathrm{D}$ space, but the resulting $2 \mathrm{D}$ boundary does not show bilateral symmetry on the $2 \mathrm{D}$ image plane due to the perspective-projection transformation, as in [36], [18], [22], [7].

The important role and use of symmetry has been studied in both human vision and computer vision [35], [41], [30], [27], [26], [11]. Particularly, prior research has shown that symmetry is nonaccidental [21], [40] and therefore can be used as a grouping cue to distinguish salient structures from noisy background. Symmetry analysis of a given object boundary is usually conducted by deriving its symmetry axis. Symmetryaxis information has been incorporated to facilitate boundary interpretation, matching, and recognition in many prior research [5], [6], [19], [42]. Note that different from the problems of boundary interpretation, matching, and recognition, the work presented in this paper aims to solve the grouping problem, where the structural boundaries are not available, and our goal is to extract them from real images.

The related work includes the long-line research on edge grouping [2], [3], [8], [9], [12], [14], [15], [23], [28], [29], [33], [37], [38], [39]. These methods aim to extract perceptually salient boundaries from a set of line segments, which are usually detected from an image by edge detectors and line-fitting operators. In previous edge-grouping methods, the grouping cost usually combines the well-known Gestalt laws such as:

1. closure, which requires the resulting boundary to be always closed,

2. proximity, which requires the gap length to be short in connecting the detected line segments into a closed boundary,

3. continuity, which requires the resulting boundary to be as smooth as possible, and

4. convexity, which requires the resulting boundary to be convex.

However, these edge-grouping methods do not consider the boundary symmetry in grouping.

Mohan and Nevatia [24] developed a grouping method, where boundary symmetry is considered along with closure and proximity. It applies both edge detection and corner detection to extract a set of line segments and corner points as the grouping tokens. The grouping cost is defined by a collinearity measure that actually reflects the proximity and continuity of the boundary. Symmetry is applied as a cue to couple the extracted curves by producing a set of ribbons. These ribbons are then grouped into structures by some heuristic algorithms. This method does not introduce a unified grouping cost function, and the developed grouping algorithm is not globally optimal. The locality of the grouping algorithm may lead to many small ribbons and may not handle the boundary occlusion very well. Another work that is closely related to the proposed work is the grouping method developed by Liu et al. [20], which identifies the local symmetry-axis segments and then applies a shortest-path algorithm to connect some of them into a complete symmetry axis. The grouping cost function is defined as the sum of a predefined local cost along the symmetry axis. By manually selecting a starting pair of points that are symmetric to each other, this method produces an open boundary. Since this method does not consider region information or other normalization in the cost function, it presents a bias toward shorter boundaries, which may have difficulty in detecting the symmetry axis shown in Fig. 1d.

In recent years, many methods have also been developed for detecting structures with symmetric appearance [25], [13], [31], [32]. For example, Prasad and Yegnanarayana [26] develop a voting-based method to detect axes of bilateral symmetry directly from images based on edge-gradient information. Note that these methods are quite different from the grouping method proposed in this paper from the following three perspectives: 1) most of these methods assume the appearance symmetry, whereas the proposed method only assumes the boundary-shape symmetry, 2) these methods usually assume the symmetry axes to be straight, whereas the proposed method quantifies symmetry as a continuous value, and 3) these methods usually detect only the symmetry axes but not the final structural boundaries, whereas the proposed method detects both symmetry axes and resulting structural boundaries.

The method proposed in this paper is inherited from the previous ratio-contour method (RC) [37], an edge-grouping method for detecting smooth closed boundaries. Particularly, both of them use the same graph algorithm: the minimumratio alternate cycle algorithm for solving the final graph problems. However, both the research goals, the problem formulations, and the graph modelings introduced in this paper are completely different from the ones introduced in [37]. The research goal in this paper is to develop a grouping method to detect boundaries with good bilateral symmetry, which is not considered in the RC. To achieve this goal, in this paper, we introduce different grouping tokens, define a different grouping cost with a normalization over the enclosed region area, construct a different graph model with "mirror" edges and auxiliary edges, and define different graph edge weight functions to encode the region-area information. In Section 5, we also compare the performance 


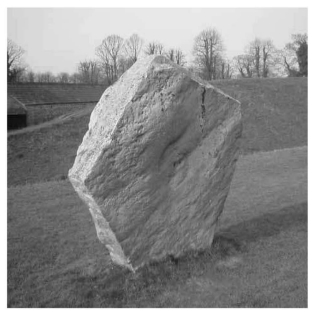

(a)

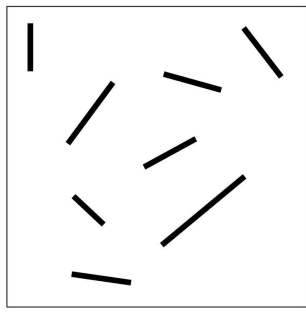

(b)

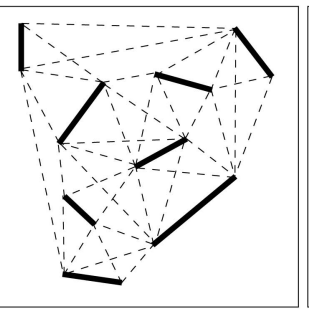

(c)

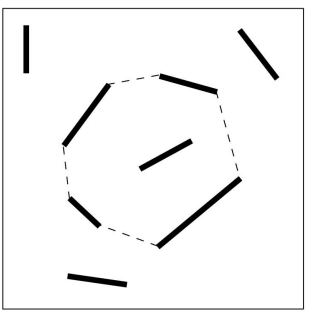

(d)

Fig. 2. An illustration of the process of edge grouping.

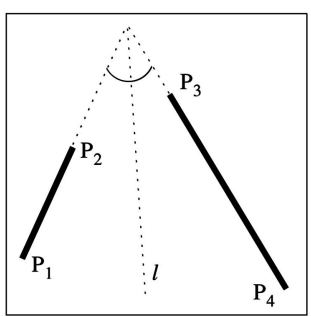

(a)

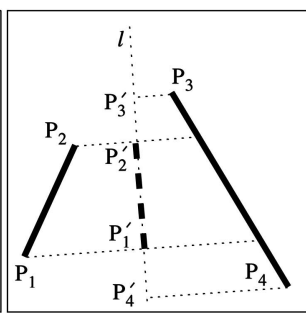

(b)

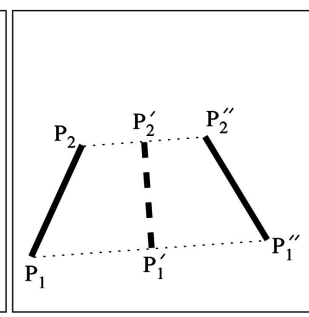

(c)

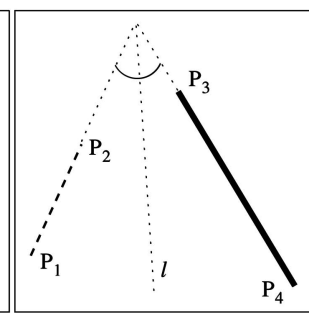

(d)

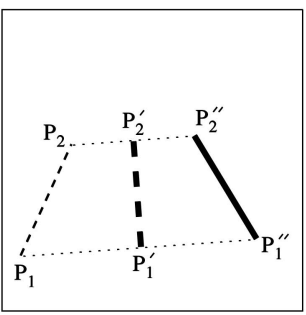

(e)

Fig. 3. An illustration of constructing a symmetric trapezoid from a pair of segments: (a), (b), and (c) pairing two detected segments and (d) and (e) pairing a gap-filling segment $P_{1} P_{2}$ with a detected segment $P_{3} P_{4}$.

of the proposed method and the RC on both synthetic data and real images.

The remainder of this paper is organized as follows: In Section 2, we formulate the problem of grouping with symmetry by introducing the general edge-grouping methodology, the new grouping tokens in the form of symmetric trapezoids, and the new unified grouping cost function. In Section 3, we introduce the graph modeling of the formulated problem and apply a graph algorithm to solve this grouping problem in a globally optimal fashion. In Section 4, we introduce a more accurate way to measure the gap length along the boundary. In Section 5, we discuss implementation details and report experimental results on both synthetic and real images. In Section 6, we discuss the possible extensions of the proposed method to incorporate other boundary or region information. In Section 7, we discuss the complexity and runtime of the proposed grouping method. Section 8 presents the conclusions.

\section{Problem Formulation}

The proposed grouping method has its roots in edge grouping, where grouping tokens are a set of line segments (or more generally, curve segments), and the output is one or several perceptually salient boundaries formed by connecting a subset of the line segments. However, to encode and quantify the boundary symmetry, we further pair the line segments to construct a new type of grouping token in the form of symmetric trapezoids. In this section, we start the problem formulation by introducing the typical process of edge grouping. We then elaborate on the trapezoid-type token construction and the grouping-cost definition.

\subsection{Edge Grouping}

In edge grouping, a set of line segments is first constructed from the input image, as shown in Fig. 2a, by edge detection and line fitting operations, as shown in Fig. $2 \mathrm{~b}$. A new set of line segments, as shown by dashed lines in Fig. 2c, is then constructed to fill the gap between each pair of initial line segments. For convenience, we call the initial line segments resulting from edge detection the detected (line) segments and the newly constructed ones the gap-filling (line) segments. A (closed) boundary of interest is then a simple cycle that traverses a set of detected and gap-filling segments alternately, as shown in Fig. $2 \mathrm{~d}$. Note that we do not show all the constructed gap-filling segments in Fig. 2c to prevent the figure from being too crowded. In the ideal case, with $n$ detected segments, we have $2 n$ segment endpoints, and therefore, we may need to construct $n(2 n-2)$ gap-filling segments if we construct a gap-filling segment between any possible two segment endpoints except for the two endpoints of the same detected segment. Finally, we define a grouping cost function for the boundaries and develop an algorithm to find from all boundaries the one with the minimum grouping cost, as shown in Fig. 2d. As mentioned above, various grouping cues such as proximity, closure, continuity, and convexity have been incorporated into edge grouping trying to extract the perceptually salient structural boundaries from a noisy background [12], [23], [28], [29], [33], [37], [38]. In prior edge-grouping methods, the grouping cost is usually defined to be a function of some local weights associated to each individual detected/gap-filling segment. However, it is difficult to incorporate symmetry into these edge-grouping methods because of the difficulty of encoding symmetry into each individual line segment. In Section 2.2, we construct new trapezoid-type tokens from these line segments to encode boundary symmetry.

\subsection{Symmetric Trapezoids as Grouping Tokens}

Although it is difficult to encode symmetry into each individual line segment, symmetry can be encoded to a pair of segments. For each pair of line segments $P_{1} P_{2}$ and $P_{3} P_{4}$, as shown in Fig. 3, we can identify their symmetric portions by following these steps: 


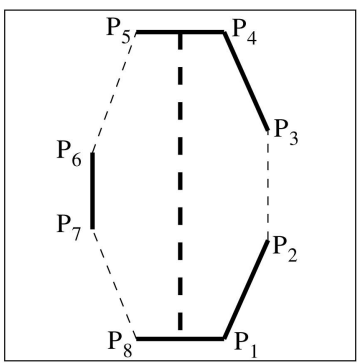

(a)

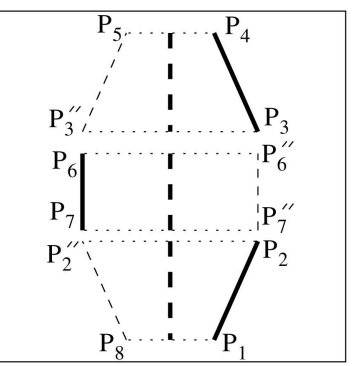

(b)
Fig. 4. An illustration of the case that needs the construction of trapezoids by pairing gap-filling segments with detected ones. (a) The desired symmetric boundary with its detected segments (solid lines), gap-filling segments (thin dashed lines), and symmetric axis (thick dashed line). (b) Trapezoids constructed by pairing gap-filling segments with detected ones.

1. Find the angle-bisector line $l$ between $P_{1} P_{2}$ and $P_{3} P_{4}$, as shown in Fig. 3a. If $P_{1} P_{2} / / P_{3} P_{4}$, then $l$ is the line equidistant to both $P_{1} P_{2}$ and $P_{3} P_{4}$.

2. Find the projections of both segments $P_{1} P_{2}$ and $P_{3} P_{4}$ to $l$ and denote them as $P_{1}^{\prime} P_{2}^{\prime}$ and $P_{3}^{\prime} P_{4}^{\prime}$, respectively. The overlap of segments $P_{1}^{\prime} P_{2}^{\prime}$ and $P_{3}^{\prime} P_{4}^{\prime}$, as shown by $P_{1}^{\prime} P_{2}^{\prime}$ in Fig. $3 \mathrm{~b}$, is denoted as the axis segment between $P_{1} P_{2}$ and $P_{3} P_{4}$. We refer to $P_{1}^{\prime}$ and $P_{2}^{\prime}$ as the axis-segment endpoints.

3. Map this axis segment back to segments $P_{1} P_{2}$ and $P_{3} P_{4}$. This results in a (symmetric) trapezoid $P_{1} P_{2} P_{2}^{\prime \prime} P_{1}^{\prime \prime}$, as shown in Fig. 3c.

In this paper, we construct such symmetric trapezoids (with axis segments) by pairing every two detected segments, as shown in Figs. 3a, 3b, and 3c, and pairing each gap-filling segment with each detected segment, as shown in Figs. 3d and 3e. Note that, for some pairs of segments, their projections to $l$ have no overlap. In this case, no symmetric trapezoid will be constructed for them. These trapezoids are used as new grouping tokens in the proposed method. We construct a trapezoid by pairing a gap-filling segment with a detected segment to handle the case shown in Fig. 4, where the desired symmetric boundary and its symmetric axis are shown in Fig. 4a. However, the symmetric portion of many detected segments such as $P_{1} P_{2}, P_{3} P_{4}$, and $P_{6} P_{7}$ are not detected and, therefore, correspond to gap-filling segments. In this case, we can represent the desired symmetric boundary only by pairing gap-filling segments with detected segments, as shown in Fig. $4 \mathrm{~b}$. For convenience, in the remainder of the paper, we usually display trapezoids by assuming that they are constructed from two detected segments and, therefore, use solid lines for both nonparallel opposite sides, as illustrated in Fig. 3c. However, we will consider both cases shown in Figs. $3 \mathrm{c}$ and $3 \mathrm{e}$ in developing the proposed grouping method.

\subsection{Trapezoid Grouping and Grouping Cost}

Analogous to edge grouping, we formulate the problem of symmetry grouping as a problem of identifying a subset of trapezoids and connecting them sequentially into a closed boundary. The gap between two sequential trapezoids in the connection is filled by a (gap-filling) quadrilateral. Two examples are shown in Figs. 5a and $5 b$, where two gap-filling quadrilaterals $\mathcal{G}_{1}=P_{2} P_{3} P_{10} P_{11}$ and $\mathcal{G}_{2}=P_{4} P_{5} P_{8} P_{9}$ are constructed to connect three trapezoids $\mathcal{T}_{1}=P_{1} P_{2} P_{11} P_{12}$, $\mathcal{T}_{2}=P_{3} P_{4} P_{9} P_{10}$, and $\mathcal{T}_{3}=P_{5} P_{6} P_{7} P_{8}$ into a closed polygonal boundary $P_{1} P_{2} \ldots P_{12}$. The axis segments of $\mathcal{T}_{1}, \mathcal{T}_{2}$, and $\mathcal{T}_{3}$ are also connected by the axis segments of the quadrilaterals $\mathcal{G}_{1}$ and $\mathcal{G}_{2}$ to generate the polyline $Q_{1} Q_{2} \ldots Q_{6}$, which is the (boundary) axis of the closed boundary $P_{1} P_{2} \ldots P_{12}$. We call $Q_{1}$ and $Q_{6}$ the boundary-axis endpoints. Note that the gap-filling quadrilaterals are simply constructed by connecting a parallel side of one trapezoid and a parallel side of another trapezoid. They may not be symmetric, and its axis segment is constructed simply by connecting the endpoints of the axis segments of the two neighboring symmetric trapezoids. As in edge grouping, in the ideal case, we may construct quadrilaterals between each pair of trapezoids. Since the axis segment of a trapezoid has two endpoints, there are four different gap-filling quadrilaterals that can be constructed between a pair of trapezoids. As discussed later, in practice, we do not need to construct all possible gap-filling quadrilaterals because many of them are not likely to be included in the desired optimal boundary.

Based on the above formulation, we can measure the symmetry of a closed boundary using the collinearity (straightness) of its boundary axis, as shown by the thick dashed polylines in Fig. 5. Specifically, we define the grouping cost function for a closed boundary $\mathcal{B}$ as

$$
\phi(\mathcal{B})=\frac{\left|\mathcal{B}_{D}\right|+\lambda \cdot \rho(\operatorname{axis}(\mathcal{B}))}{\operatorname{area}(\mathcal{B})},
$$

where $\left|\mathcal{B}_{D}\right|$ is the total gap length along the boundary $\mathcal{B}$. Note that not only quadrilaterals but also trapezoids may contribute to $\left|\mathcal{B}_{D}\right|$, because 1) trapezoids may be constructed by pairing a gap-filling segment with a detected segment, as shown in Figs. 3d and 3e, and 2) a parallel side of a trapezoid may contribute to the boundary gap-length $\left|\mathcal{B}_{D}\right|$ when this

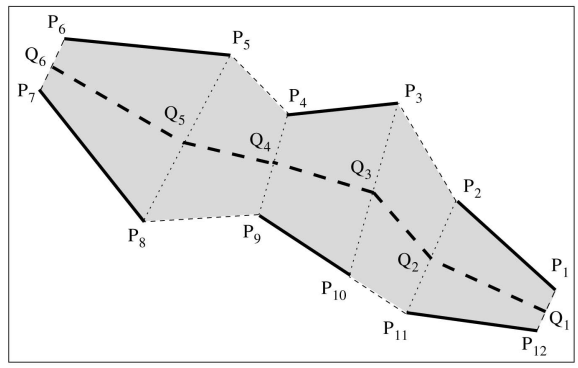

(a)

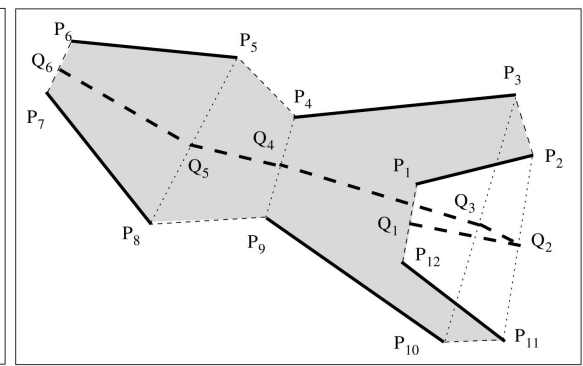

(b)

Fig. 5. Two examples of grouping detected trapezoids into a closed boundary. 


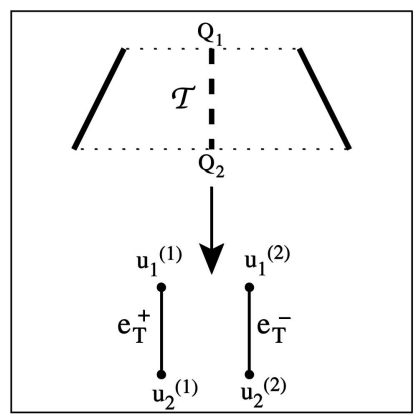

(a)

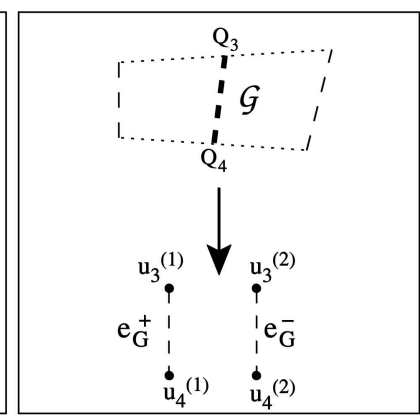

(b)
Fig. 6. An illustration of the solid/dashed edges. (a) For a trapezoid $\mathcal{T}$, we construct a pair of solid edges $e_{T}^{+}$and $e_{T}^{-}$. Each axis-segment endpoint $Q_{i}$ is then modeled by two vertices $u_{i}^{(1)}$ and $u_{i}^{(2)}$. (b) For a gapfilling quadrilateral $\mathcal{G}$, we proceed similarly as in (a), except that the two constructed edges are dashed.

side contains a boundary-axis endpoint, for example, the parallel sides $P_{1} P_{12}$ and $P_{6} P_{7}$ need to be included in calculating $\left|\mathcal{B}_{D}\right|$ in Fig. 5. In Section 4, we will further discuss a more accurate way to measure $\left|\mathcal{B}_{D}\right|$ in practice. The term of $\left|\mathcal{B}_{D}\right|$ reflects the preference of a boundary with good proximity. The term of $\rho(\operatorname{axis}(\mathcal{B}))$ is a measure related to the collinearity of $\mathcal{B}^{\prime}$ s axis (for example, the thick dashed polylines in Fig. 5). This term reflects the preference of a boundary with good symmetry, and we will elaborate on this term in Section 3. The term of $\operatorname{area}(\mathcal{B})$ is the region area enclosed by the boundary $\mathcal{B}$. A normalization over this term sets a preference to produce larger rounder structures, which improves the robustness against image noise by avoiding a bias toward shorter boundaries. Note that, in practice, such a preference may not prevent the detection of small salient structures if they show good proximity and symmetry, as we will see in many experiments reported in Section 5. $\lambda>0$ is a preset factor that balances the weights of the proximity and symmetry terms. As discussed later, we simply set a consistent $\lambda=10$ in all the experiments reported in this paper.

\section{Graph Modeling and Algorithm}

In this section, we construct a new graph model to describe the above formulated problem. In this graph model, trapezoids and gap-filling quadrilaterals are represented by graph edges. By encoding the grouping cost (1) into the graph edge weights, we then reduce the grouping problem to a problem of finding an optimal cycle with minimum ratio cost in this new graph. We finally apply a known graph algorithm to address this cycle-finding problem. Specifically, the graph construction consists of two sequential steps: 1) constructing solid and dashed edges to represent trapezoids and gap-filling quadrilaterals, respectively, and 2) further constructing auxiliary edges between the vertices corresponding to potential axis endpoints. We elaborate on these two steps as follows.

\subsection{Graph Construction I: Solid/Dashed Edges}

We construct an undirected graph $G=(V, E)$ with vertex set $V$ and edge set $E$ to model the trapezoids and gap-filling quadrilaterals. To encode the enclosed region area area $(\mathcal{B})$, we construct a pair of solid edges $e_{T}^{+}$and $e_{T}^{-}$for each trapezoid $\mathcal{T}$, and a pair of dashed edges $e_{G}^{+}$and $e_{G}^{-}$for each quadrilateral $\mathcal{G}$, as shown in Fig. 6. We call the pair of the

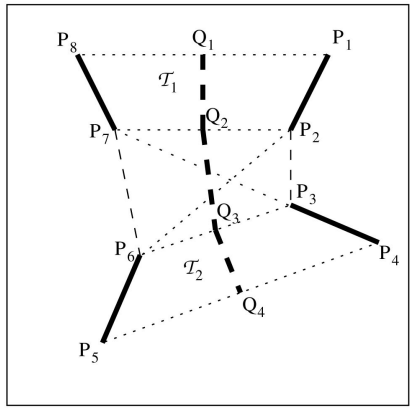

Fig. 7. An illustration of determining the gap-filling quadrilateral between two trapezoids $\mathcal{T}_{1}=P_{1} P_{2} P_{7} P_{8}$ and $\mathcal{T}_{2}=P_{3} P_{4} P_{5} P_{6}$.

edges constructed for the same trapezoid or quadrilateral to be the mirror edges of each other. For convenience, we can treat each pair of mirror edges to be an abstraction of the axis segment of the corresponding trapezoid or quadrilateral. For example, $e_{T}^{+}$and $e_{T}^{-}$model the axis segment $Q_{1} Q_{2}$ in Fig. 6a. Accordingly, we construct a pair of vertices for each axis-segment endpoint (or each parallel side of each trapezoid). For example, vertex pair $u_{1}^{(1)}$ and $u_{1}^{(2)}$ are constructed for axis-segment endpoint $Q_{1}$ in Fig. 6a.

In the proposed grouping, two neighboring trapezoids are connected by a quadrilateral, as shown in Fig. 5. Therefore, in constructing the graph $G=(V, E)$, we connect two pairs of solid edges by a pair of dashed edges, since each trapezoid and quadrilateral is represented by two mirror edges $e^{+}$and $e^{-}$. We apply the following two steps to determine the edge connection in constructing the graph $G=(V, E)$. First, we consider only the quadrilateral that leads to a nonintersected boundary. Particularly, when connecting two trapezoids by a quadrilateral, the two sides of the selected quadrilateral that are not shared with the two trapezoids must not intersect with each other. For example, in Fig. 7, we construct the quadrilateral in the form of $P_{2} P_{3} P_{6} P_{7}$ with two sides $P_{2} P_{3}$ and $P_{6} P_{7}$, instead of the quadrilateral $P_{2} P_{6} P_{3} P_{7}$ with two sides $P_{2} P_{6}$ and $P_{3} P_{7}$ to connect $\mathcal{T}_{1}$ and $\mathcal{T}_{2}$.

Second, we distinguish two mirror edges $e^{+}$and $e^{-}$by associating an implicit direction to the corresponding trapezoid or quadrilateral. Particularly, we set edge $e^{+}$to imply that its corresponding trapezoid or quadrilateral has a counterclockwise direction and edge $e^{-}$to imply that its corresponding trapezoid or quadrilateral has a clockwise direction. Then, the edge connection will be uniquely determined by requiring the resulting boundary to be of a consistent direction, either clockwise or counterclockwise. Figs. $8 \mathrm{a}, 8 \mathrm{~b}, 8 \mathrm{c}$, and $8 \mathrm{~d}$ illustrate the four possible cases. When the clockwise $\mathcal{T}_{1}$ is connected to the clockwise $\mathcal{T}_{2}$ by a gap-filling quadrilateral (this implies that the counterclockwise $\mathcal{T}_{1}$ is connected to the counterclockwise $\mathcal{T}_{2}$ ), we connect their corresponding edges with same signs, as shown in Figs. 8a and 8c. When the clockwise $\mathcal{T}_{1}$ is connected to the counterclockwise $\mathcal{T}_{2}$ by a gap-filling quadrilateral (this implies that the counterclockwise $\mathcal{T}_{1}$ is connected to the clockwise $\mathcal{T}_{2}$ ), we connect their corresponding edges with opposite signs, as shown in Figs. 8b and $8 \mathrm{~d}$. The sign of the edge corresponding to the quadrilateral can also be uniquely determined by following the directions of the trapezoids. For example, in Fig. 8a, the quadrilateral between the clockwise $\mathcal{T}_{1}$ and the clockwise $\mathcal{T}_{2}$ has a clockwise direction. This indicates that 


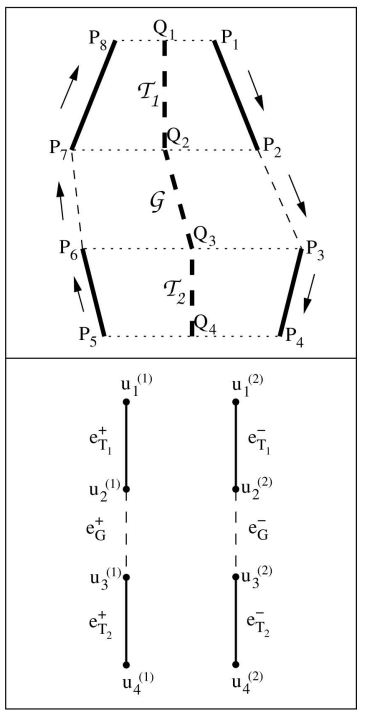

(a)

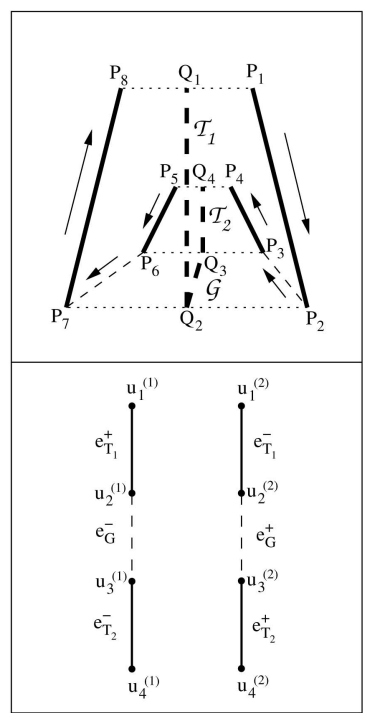

(b)

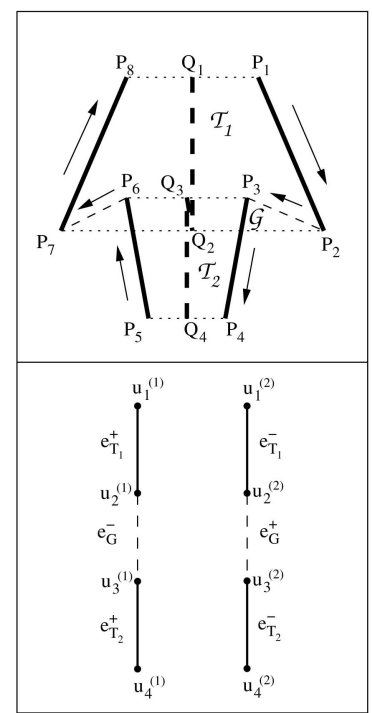

(c)

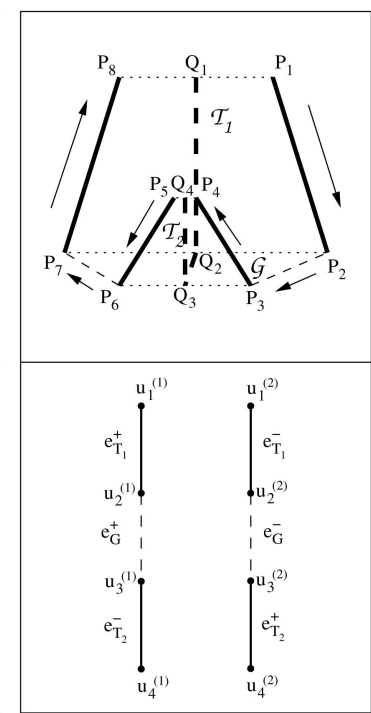

(d)

Fig. 8. An illustration of the edge connection for modeling the construction of a quadrilateral between two trapezoids.

the dashed edge between $e_{T_{1}}^{+}$and $e_{T_{2}}^{+}$is $e_{G}^{+}$, and the dashed edge between $e_{T_{1}}^{-}$and $e_{T_{2}}^{-}$is $e_{G}^{-}$.

There is a special case where the constructed gap-filling quadrilateral contains a self intersection, as shown by the quadrilateral $P_{2} P_{3} P_{6} P_{7}$ in Fig. 9. Note that this self intersection is caused by the two sides $P_{3} P_{6}$ and $P_{2} P_{7}$ from the two neighboring trapezoids and will not be included in the resulting boundary. Therefore, different from the quadrilateral self intersection mentioned before, this kind of selfintersected quadrilateral is allowed as it does not lead to selfintersected boundaries. In this case, the quadrilateral is divided into two triangles $R_{1}$ and $R_{2}$ with opposite directions, that is, if $R_{1}$ is counterclockwise, then $R_{2}$ is clockwise, also as shown in Fig. 9. We set this quadrilateral's direction to be the direction of the triangle with a larger area and set the area of this quadrilateral as $\left|\operatorname{area}\left(R_{1}\right)-\operatorname{area}\left(R_{2}\right)\right|$.

For each edge $e$ in this graph, we define two weight functions $w_{1}(e)$ and $w_{2}(e)$. If $e$ is solid, we define $w_{1}(e)=0$ if the corresponding trapezoid was constructed from two detected segments, for example, the trapezoid shown in Fig. 3c; otherwise, we set $w_{1}(e)$ to be the length of the gapfilling segment included in the trapezoid construction, for example, $\left|P_{1} P_{2}\right|$, the Euclidean distance between $P_{1}$ and $P_{2}$, in Fig. 3e. If $e$ is dashed, for example, corresponding to axis segment $Q_{2} Q_{3}$ in Fig. 7, we define

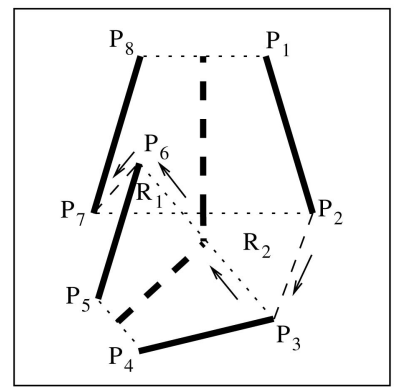

Fig. 9. An illustration of an allowed self-intersected quadrilateral.

$$
w_{1}(e)=\left|P_{2} P_{3}\right|_{D}+\left|P_{6} P_{7}\right|_{D}+\lambda \cdot \rho(e),
$$

where $\left|P_{2} P_{3}\right|_{D}+\left|P_{6} P_{7}\right|_{D}$ is the total gap length (along the boundary) that results from the quadrilateral corresponding to $e$. In this paper, we use an improved gap-length measure $\left|P_{i} P_{j}\right|_{D}$ instead of the Euclidean distance $\left|P_{i} P_{j}\right|$ to handle the case where a gap-filling quadrilateral may (partially) coincide with detected segments. We will discuss this improved gap-length measure in Section 4. $\rho(e)=\left|\sin \left(\angle Q_{1} Q_{2} Q_{4}\right)\right|+$ $\left|\sin \left(\angle Q_{1} Q_{3} Q_{4}\right)\right|$ measures the collinearity (straightness) of the polyline $Q_{1} Q_{2} Q_{3} Q_{4}$, giving a lower cost to polylines with good collinearity, as shown in Fig. 7. We can see that the first edge weight $w_{1}(e)$ is always nonnegative and for a pair of mirror edges $e^{+}$and $e^{-}$, we have $w_{1}\left(e^{+}\right)=w_{1}\left(e^{-}\right)$. The second edge weight, $w_{2}(e)$, is simply the signed area of the trapezoid or quadrilateral corresponding to $e$, that is, $w_{2}\left(e^{+}\right)=-w_{2}\left(e^{-}\right)>0$. We introduce the negative area to handle the nonconvex boundaries, where the inclusion of an additional trapezoid or quadrilateral may contribute negatively to the resulting enclosed $\operatorname{area} \operatorname{area}(\mathcal{B})$, as shown by the trapezoid $P_{1} P_{2} P_{11} P_{12}$ in Fig. 5b.

\subsection{Graph Construction II: Auxiliary Edges}

In order to include the boundary information around the two boundary-axis endpoints, for example, the gap length along $P_{6} P_{7}$ and $P_{1} P_{12}$ in Fig. 5, we further construct a set of new dashed edges, named auxiliary edges, between the vertices corresponding to the two boundary-axis endpoints. Let $u_{1}^{(1)}$ and $u_{1}^{(2)}$ be the vertex pair corresponding to a boundaryaxis endpoint, for example, $Q_{1}$ in Fig. 5a (or Fig. 5b), and let $u_{6}^{(1)}$ and $u_{6}^{(2)}$ be the vertex pair corresponding to the other boundary-axis endpoint, for example, $Q_{6}$ in Fig. 5a (or Fig. 5b). We construct four dashed edges $\left(u_{1}^{(i)}, u_{6}^{(j)}\right), i, j=$ 1,2 as auxiliary edges, as shown by dashed-dotted curves in Fig. 10a (or Fig. 10b). For these four auxiliary edges, we set their second edge weights to be zero and the first edge weights to be the total gap length around these two boundary-axis endpoints. For example, in Fig. 5a (or Fig. 5b), the first weight $w_{1}(e)$ of the four auxiliary edges that reflects the connection between $Q_{1}$ and $Q_{6}$ is defined by 


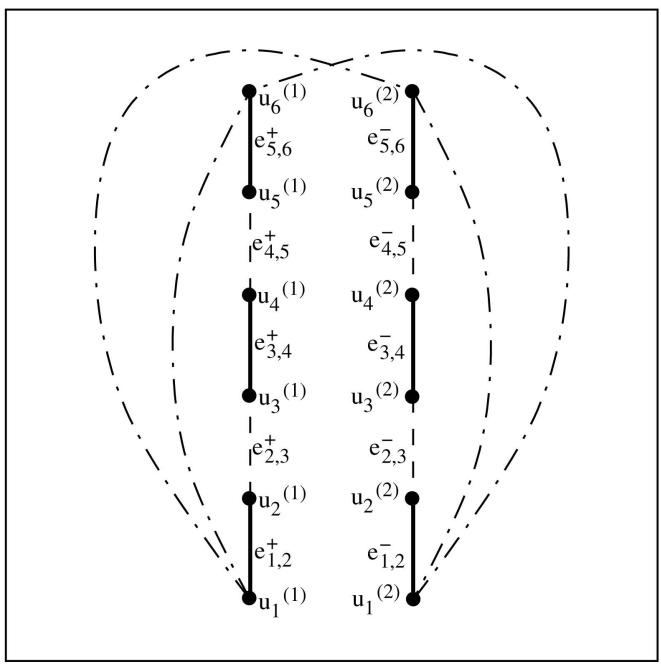

(a)

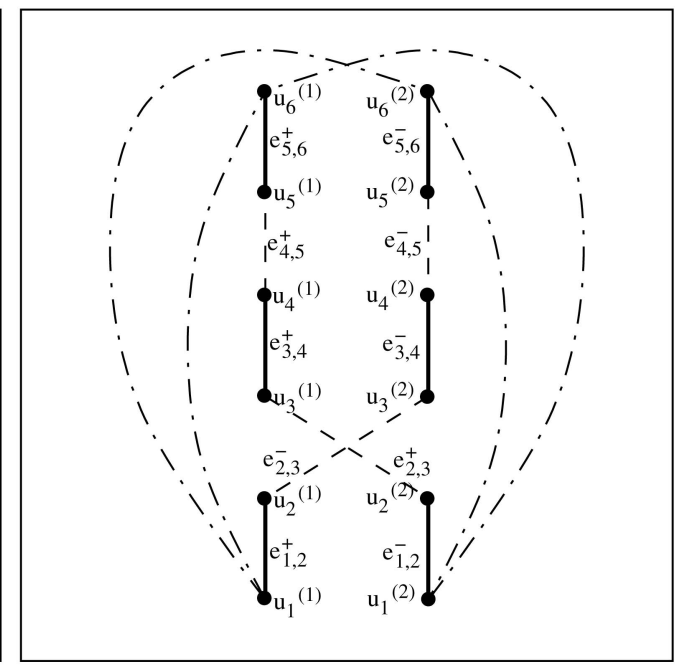

(b)

Fig. 10. An illustration of the cycles corresponding to the boundaries shown in Figs. $5 a$ and $5 b$, respectively. (a) For the boundary in Fig. $5 a$, the corresponding cycle $\mathcal{C}^{+}$traverses $u_{i}^{(1)}, i=1,2, \ldots, 6$ sequentially and $\mathcal{C}^{-}$traverses $u_{i}^{(2)}, i=1,2, \ldots, 6$ sequentially. (b) For the boundary shown in Fig. $5 \mathrm{~b}$, the corresponding cycle $\mathcal{C}^{+}$traverses $u_{1}^{(2)}, u_{2}^{(2)}, u_{3}^{(1)}, u_{4}^{(1)}, u_{5}^{(1)}$, and $u_{6}^{(1)}$ sequentially, and $\mathcal{C}^{-}$traverses $u_{1}^{(1)}, u_{2}^{(1)}, u_{3}^{(2)}, u_{4}^{(2)}, u_{5}^{(2)}$, and $u_{6}^{(2)}$ sequentially. Vertices $u_{i}^{(1)}$ and $u_{i}^{(2)}$ correspond to the axis-segment endpoint $Q_{i}, i=1,2, \ldots, 6$ in Fig. 5. Auxiliary edges are shown by dashed-dotted curves.

$$
w_{1}(e)=\left|P_{1} P_{12}\right|_{D}+\left|P_{6} P_{7}\right|_{D},
$$

where $\left|P_{i} P_{j}\right|_{D}$ is the gap length between $P_{i}$ and $P_{j}$ to be detailed in Section 4. Since the optimal closed boundary and its boundary-axis endpoints are unknown before the grouping, we can treat all axis-segment endpoints as potential boundary-axis endpoints and construct auxiliary edges between all of them. In practice, however, we do not need to construct auxiliary edges between all axis-segment endpoints, and we will discuss this in more detail in Section 5.

In the graph $G=(V, E)$, each boundary $\mathcal{B}$ is represented by two "mirror" cycles $\mathcal{C}^{+}$and $\mathcal{C}^{-}$, for example, if an edge is included in $\mathcal{C}^{+}$, its mirror edge must be included in $\mathcal{C}^{-}$, and vice versa. In addition, each of them contains an auxiliary edge, as shown in Fig. 10. These two cycles traverse a sequence of solid and dashed edges alternately, and therefore, we call them alternate cycles. It is easy to verify that 1 ) the total first edge weights along both $\mathcal{C}^{+}$and $\mathcal{C}^{-}$are always equal to the numerator of the cost $\phi(\mathcal{B})$ in (1) and 2) the total second edge weights along $\mathcal{C}^{+}$and $\mathcal{C}^{-}$have the same absolute value equal to $\operatorname{area}(\mathcal{B})$ but with opposite signs. Without loss of generality, we assume $\sum_{e \in \mathcal{C}^{+}} w_{2}(e)=-\sum_{e \in \mathcal{C}^{-}} w_{2}(e)>0$. This way, we have

$$
\phi(\mathcal{B})=\frac{\sum_{e \in \mathcal{C}^{+}} w_{1}(e)}{\sum_{e \in \mathcal{C}^{+}} w_{2}(e)}=-\frac{\sum_{e \in \mathcal{C}^{-}} w_{1}(e)}{\sum_{e \in \mathcal{C}^{-}} w_{2}(e)}
$$

and locating the optimal boundary $\mathcal{B}$ that minimizes the cost (1) is reduced to finding an alternate cycle $\mathcal{C}$ in graph $G=$ $(V, E)$ such that this cycle $\mathcal{C}$ minimizes the cycle ratio

$$
\varphi(\mathcal{C})=\frac{W_{2}(\mathcal{C})}{W_{1}(\mathcal{C})}=\frac{\sum_{e \in \mathcal{C}} w_{2}(e)}{\sum_{e \in \mathcal{C}} w_{1}(e)} .
$$

The correctness of this reduction comes from the following two facts: First, any alternate cycle $\mathcal{C}$ has two mirror versions $\mathcal{C}^{+}$and $\mathcal{C}^{-}$with opposite signs on the total second weights. Clearly, the cycle $\mathcal{C}$ that minimize the cost (2) must be of a $\mathcal{C}^{-}$version and has a negative $W_{2}(\mathcal{C})$. The mirror of this optimal cycle $\mathcal{C}$ is then of a $\mathcal{C}^{+}$version that maximizes
$\frac{W_{2}(\mathcal{C})}{W_{1}(\mathcal{C})}$ and, therefore, minimizes $\frac{W_{1}(\mathcal{C})}{W_{2}(\mathcal{C})}$ subject to $W_{2}(\mathcal{C})>0$. The ratio $\frac{W_{1}(\mathcal{C})}{W_{2}(\mathcal{C})}$ subject to $W_{2}(\mathcal{C})>0$ is exactly the same as $\phi(\mathcal{B})$, where area $(\mathcal{B})$ is always positive. Therefore, we have $\phi(\mathcal{B})=-\frac{1}{\varphi(\mathcal{C})}$ and the alternate cycle $\mathcal{C}$ with the minimum cycle ratio $\varphi(\mathcal{C})$ corresponds to the boundary $\mathcal{B}$ with the minimum cost $\phi(\mathcal{B})$.

Second, we prove by contradiction that the resulting optimal alternate cycle $\mathcal{C}$ does not contain more than one auxiliary edge. Otherwise, the resulting boundary $\mathcal{B}$ would contain multiple separate closed boundaries with unaligned axes. Assume the resulting cycle $\mathcal{C}$ contains $k$ auxiliary edges with $k>1$. This means $\mathcal{C}$ contains $k$ alternate paths $\mathcal{P}_{1}, \mathcal{P}_{2}, \ldots, \mathcal{P}_{k}$ after these $k$ auxiliary edges are removed. From these $k$ paths, we can construct $k$ new alternate cycles $\mathcal{C}_{1}, \mathcal{C}_{2}, \ldots, \mathcal{C}_{k}$ by including the auxiliary edges between the two endpoints of each path. Given the cycle ratio defined in (2), it is not difficult to see that

$$
\varphi(\mathcal{C})=\frac{W_{2}(\mathcal{C})}{W_{1}(\mathcal{C})}=\frac{\sum_{i=1}^{k} W_{2}\left(\mathcal{C}_{i}\right)}{\sum_{i=1}^{k} W_{1}\left(\mathcal{C}_{i}\right)} .
$$

This shows that at least one cycle, say $\mathcal{C}_{m}$, out of $\mathcal{C}_{i}$, $i=1,2, \ldots, k$, has a smaller cycle ratio than $\mathcal{C}$, that is, $\varphi\left(\mathcal{C}_{m}\right)=\frac{W_{2}\left(\mathcal{C}_{m}\right)}{W_{1}\left(\mathcal{C}_{m}\right)} \leq \varphi(\mathcal{C})$. This contradicts the assumption that $\mathcal{C}$ is a cycle with minimum cycle ratio.

Finally, we use an available graph algorithm to find an alternate cycle $\mathcal{C}$ that minimizes the cycle ratio (2) [37], [1]. This minimum-ratio alternate cycle algorithm finds the desired optimal cycle in polynomial time.

\section{An Improved Gap-Length Measure $\left|P_{i} P_{j}\right|_{D}$}

The main goal of introducing $\left|P_{i} P_{j}\right|_{D}$ is to handle the case where a gap-filling quadrilateral may coincide with detected segments. As shown in both Figs. 11a and 11b, a gap-filling quadrilateral $P_{2} P_{3} P_{6} P_{7}$ is constructed to connect two trapezoids $\mathcal{T}_{1}$ and $\mathcal{T}_{2}$. The contribution of this quadrilateral to the term $\left|\mathcal{B}_{D}\right|$, if it is included in the boundary $\mathcal{B}$, is not 


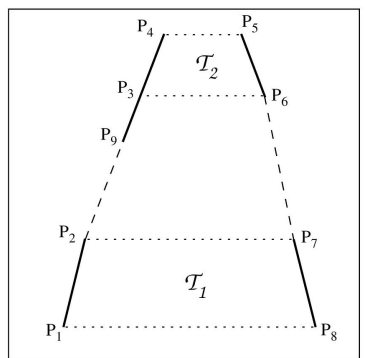

(a)

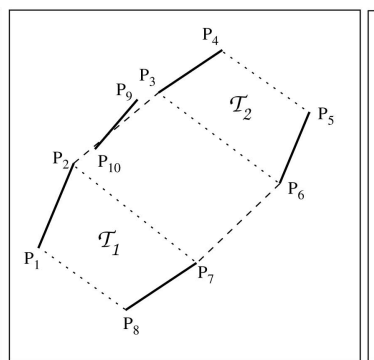

(b)

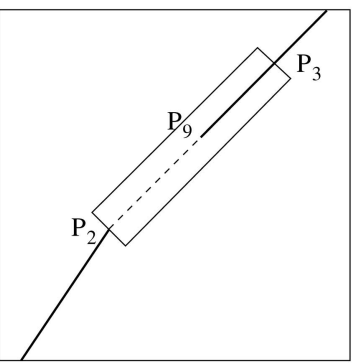

(c)

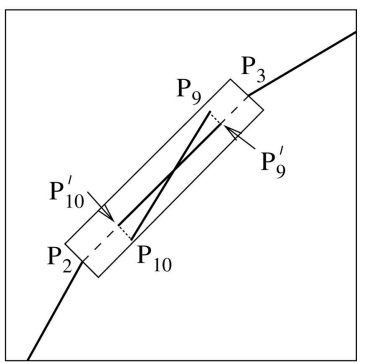

(d)

Fig. 11. An illustration of measuring the gap length by excluding the projections from coincident detected segments: (a) and (b) two examples where the side $P_{2} P_{3}$ of the quadrilateral $P_{2} P_{3} P_{6} P_{7}$ is coincident with a detected segment, and (c) and (d) calculating $\left|P_{2} P_{3}\right|_{D}$ for the two examples shown in (a) and (b), respectively.

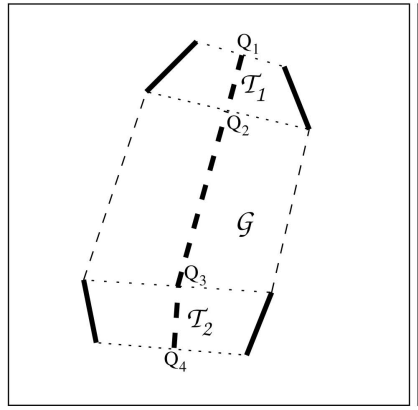

(a)

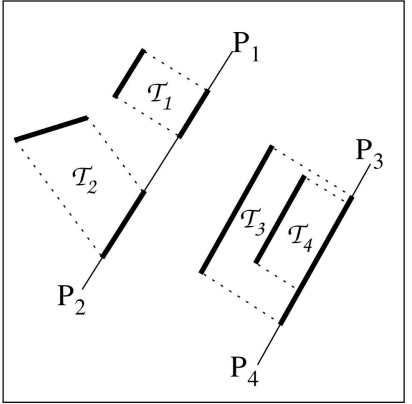

(b)

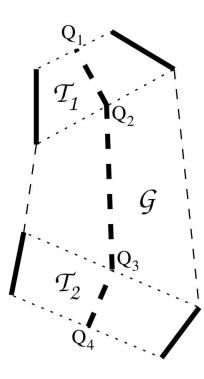

(c)

Fig. 12. An illustration of the three strategies implemented to reduce the number of quadrilaterals.

$\left|P_{2} P_{3}\right|+\left|P_{6} P_{7}\right|$, as a portion of $P_{2} P_{3}$ is in fact coincident with detected segments, for example, $P_{3} P_{9}$ in Fig. 11a and $P_{9} P_{10}$ in Fig. 11b. To get an accurate estimate of $\left|\mathcal{B}_{D}\right|$, we locate such coincident portions and deduct them from the calculation of the gap length.

Specifically, in calculating the gap length between two points, say, $P_{2}$ and $P_{3}$ in Figs. 11c and 11d, we construct a rectangular box, shown in Figs. $11 \mathrm{c}$ and $11 \mathrm{~d}$, around the segment $P_{2} P_{3}$. All the detected segments, for example, $P_{9} P_{10}$ in Fig. 11d or the portions of the detected segments, for example, $P_{9} P_{3}$ in Fig. 11c, that are located inside of this rectangular box are projected onto the segment $P_{2} P_{3}$. We then only count the portions along $P_{2} P_{3}$ that do not overlap with any such detected-segment projections to calculate the gaplength between $P_{2}$ and $P_{3}$. For example, the gap length between $P_{2} P_{3}$ will be $\left|P_{2} P_{3}\right|_{D}=\left|P_{2} P_{9}\right|$ for the case shown in Fig. 11c and $\left|P_{2} P_{3}\right|_{D}=\left|P_{2} P_{10}^{\prime}\right|+\left|P_{3} P_{9}^{\prime}\right|$ for the case shown in Fig. 11d. Using a small rectangular box for searching coincident detected segments improves the robustness by not requiring exact coincidence between the considered gap and the detected segments, as shown in Fig. 11d. The only free parameter in this processing is the width of the rectangular box. In all our experiments, we set this width to be 2 pixels.

\section{EXPERIMENTS}

We implemented the proposed method and tested its performance on synthetic data and on a set of real images. ${ }^{1}$ For the synthetic data, we synthesize detected segments directly, and all the detected segments are located within a

1. The software and images used in this section are available at http:// www.cse.sc.edu/ songwang/document/SRC.tgz. square region of size $96 \times 96$. All the real images are scaled to be no larger than $250 \times 250$ while maintaining their aspect ratio. For all our experiments, we set $\lambda=10$ in the cost function (1). We will discuss the selection of this parameter later in Section 5.4.

In constructing the trapezoids, we pair 1) every two detected segments and 2) every gap-filling segment with every detected segment, when they have overlapped projections on their angle-bisector line, as shown in Fig. 3. For the gap-filling segments, we consider no more than $K$ shortest gap-filling segments incident from each detected-segment endpoints, where $K$ is a preset constant number, and we set $K=5$ in our experiments. This way, we consider only $O(n)$ gap-filling segments out of all possible $O\left(n^{2}\right)$ ones with $n$ being the number of detected segments. This indicates that we may construct $O\left(n^{2}\right)$ trapezoids. If we construct quadrilaterals to fill the gaps between each pair of trapezoids, we may then have $O\left(n^{4}\right)$ quadrilaterals, which lead to $O\left(n^{4}\right)$ dashed edges in the constructed graph. To reduce the number of dashed edges, we develop three practical strategies to avoid constructing the quadrilaterals that are unlikely to be included in the desired optimal boundary.

The first strategy is to consider the construction of only one quadrilateral between each pair of trapezoids. As discussed above, we can construct four different gap-filling quadrilaterals to connect two trapezoids because the axis segment of a trapezoid has two endpoints. For example, to connect the two trapezoids shown in Fig. 12a, the axissegment of the constructed quadrilateral can be $Q_{1} Q_{3}$, $Q_{1} Q_{4}, Q_{2} Q_{3}$, or $Q_{2} Q_{4}$. Usually, only one of these four quadrilaterals is likely to be included in the desired optimal boundary. Based on the proximity preference, we only consider the quadrilateral that has the shortest axis segment 


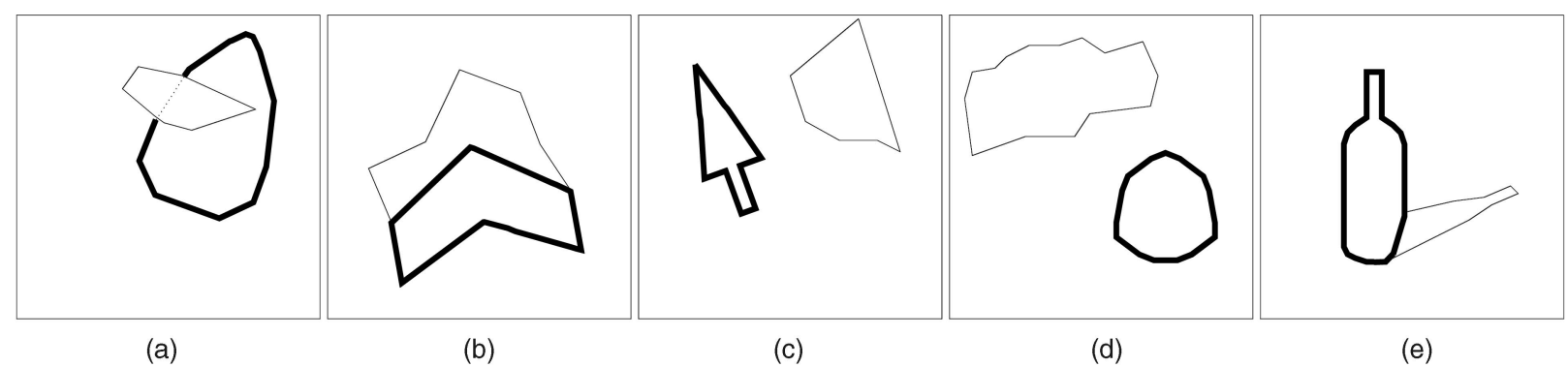

Fig. 13. Five boundary pairs used for constructing synthetic data. Desired symmetric boundaries are shown in bold.

out of the four choices. For the example shown in Fig. 12a, we only consider one quadrilateral with the axis segment $Q_{2} Q_{3}$ to connect the trapezoids $\mathcal{T}_{1}$ and $\mathcal{T}_{2}$. Furthermore, if the considered quadrilateral introduces a total gap length that is larger than a given threshold value, we simply do not construct it. In our experiments, we set this threshold value to $D_{1}=30$ pixels. Each quadrilateral introduces two gaps described by its two sides across the two neighboring trapezoids, for example, the gap length introduced by the quadrilateral $P_{2} P_{3} P_{6} P_{7}$ in Fig. 11a (or Figs. 11b) is $\left|P_{2} P_{3}\right|_{D}+\left|P_{6} P_{7}\right|_{D}$.

Our second strategy is to avoid constructing a quadrilateral to connect two trapezoids that share a same portion of a detected segment. For example, the two trapezoids $\mathcal{T}_{3}$ and $\mathcal{T}_{4}$ shown in Fig. $12 \mathrm{~b}$ share a same portion of the detected segment $P_{3} P_{4}$. Therefore, no quadrilateral will be constructed between them since the resulting closed boundary should not traverse (a portion of) a line segment more than once. However, for two trapezoids $\mathcal{T}_{1}$ and $\mathcal{T}_{2}$ shown in Fig. 12b, although both of them are constructed from the detected segment $P_{1} P_{2}$, we still consider constructing a quadrilateral to connect them since they use different portions of $P_{1} P_{2}$.

Our third strategy is to avoid constructing quadrilaterals that lead to an axis with low collinearity, because we are only interested in detecting symmetric boundaries. Specifically, for the two trapezoids shown in Fig. 12c, we require that $\left|\sin \left(\angle Q_{1} Q_{2} Q_{4}\right)\right|$ and $\left|\sin \left(\angle Q_{1} Q_{3} Q_{4}\right)\right|$ are both less than a certain threshold for constructing a quadrilateral between them. These two terms are exactly the ones used in the grouping cost (1) for measuring the local boundary symmetry. In our experiments, we set this threshold to $D_{2}=0.5$. Applying these three strategies can substantially reduce the number of dashed edges in the constructed graph.

Similarly, the number of the auxiliary edges would be $O\left(n^{4}\right)$ if we consider connecting every possible pair of the axis-segment endpoints. To reduce the number of auxiliary edges, we only consider the axis-segment endpoints that are likely to be boundary-axis endpoints for constructing auxiliary edges. Specifically, we only consider the axissegment endpoints around which the gap length is less than a given threshold. For example, in Fig. 5a, we may consider $Q_{1}$ as a potential boundary-axis endpoint for constructing auxiliary edges because the gap length between $P_{1}$ and $P_{12}$ is small. However, we may not consider $Q_{5}$ because the gap length between $P_{5}$ and $P_{8}$ is larger than the given threshold. In our experiments, we set this threshold to $D_{3}=20$ pixels. In addition, if both endpoints of a trapezoid axis segment satisfy this condition to be potential boundary-axis endpoints, we only consider the one with the smaller gap length.
Note that the gap length is measured by the method introduced in Section 4.

Note that all these strategies are developed for speeding up the algorithm in practice. Without these three strategies, the number of constructed edges in the graph is still a polynomial function of $n$. Therefore, the complexity of the proposed grouping algorithm is still polynomial in terms of $n$. The robustness of the method to these thresholds is examined in Section 5.4 .

\subsection{Experiments on Synthetic Data}

To evaluate the proposed method quantitatively, we construct a set of synthetic data with a known ground truth of the desired symmetric boundary. Each synthetic data sample is constructed in the form of a set of detected segments, which come from two sources: a pair of synthetic boundaries (one desired symmetric boundary and one nonsymmetric boundary) and random noise. Fig. 13 shows the boundary pairs that are used for constructing synthetic data. The desired symmetric boundaries are shown in bold. We can see that the pair of boundaries in Fig. 13 may or may not overlap each other since both cases may happen in practice. Particularly, Fig. 13e simulate the case where a symmetric structure generates a nonsymmetric shadow. In some cases, such as the ones shown in Fig. 13d, the desired symmetric boundary encloses a smaller area than the other nonsymmetric boundary does. We intentionally design such cases to see whether the consideration of the boundary symmetry can help detect the desired symmetric boundary, even if the grouping cost prefers a boundary with a larger enclosed region area.

We sample the boundaries shown in Fig. 13 to construct disjoint detected segments with gaps. Specifically, we uniformly subdivide each boundary into a set of line segments of equal length (5 percent of the perimeter) and then randomly remove a certain number of these line segments. The remaining ones are then included as detected segments in constructing a synthetic data sample. We further add randomly generated detected segments to simulate the image noise and for simplicity, we call them noise segments. For these noise segments, their directions conform to a uniform distribution over all possible directions, their locations conform to a uniform distribution within a square region of size $96 \times 96$, and their lengths are uniformly distributed within the range of $[5.0,15.0]$ pixels. Fig. 14a shows an example of adding 40 noise segments to the boundary pair shown in Fig. 13a. Fig. 14b shows one synthetic data sample, which consists of the detected segments sampled from the boundary pair shown in Fig. 13a and the noise segments shown in Fig. 14a. Particularly, we remove 


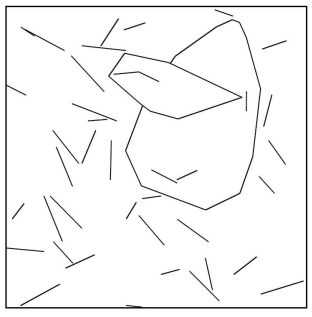

(a)

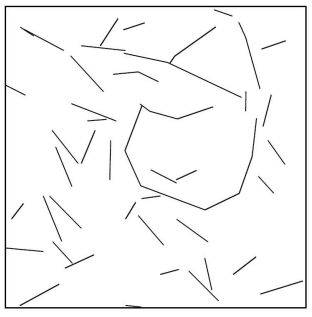

(b)

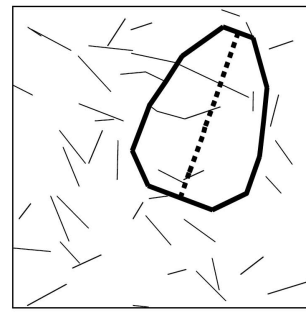

(c)

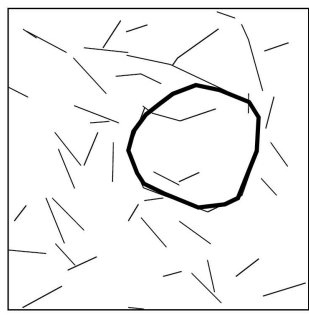

(d)

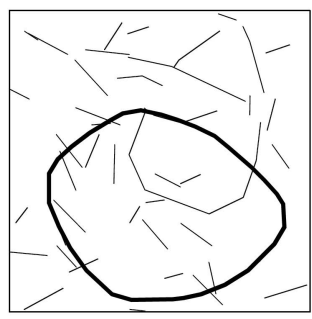

(e)

Fig. 14. An illustration of a synthetic data sample. (a) A boundary pair and random noise segments. (b) Synthesized detected segments by combining the ones from the boundary pairs and the noise segments. (c) Optimal boundary detected by the proposed grouping method with symmetry axis shown as a dashed curve. (d) Optimal boundary detected by RC. (e) Optimal boundary detected by EZ.

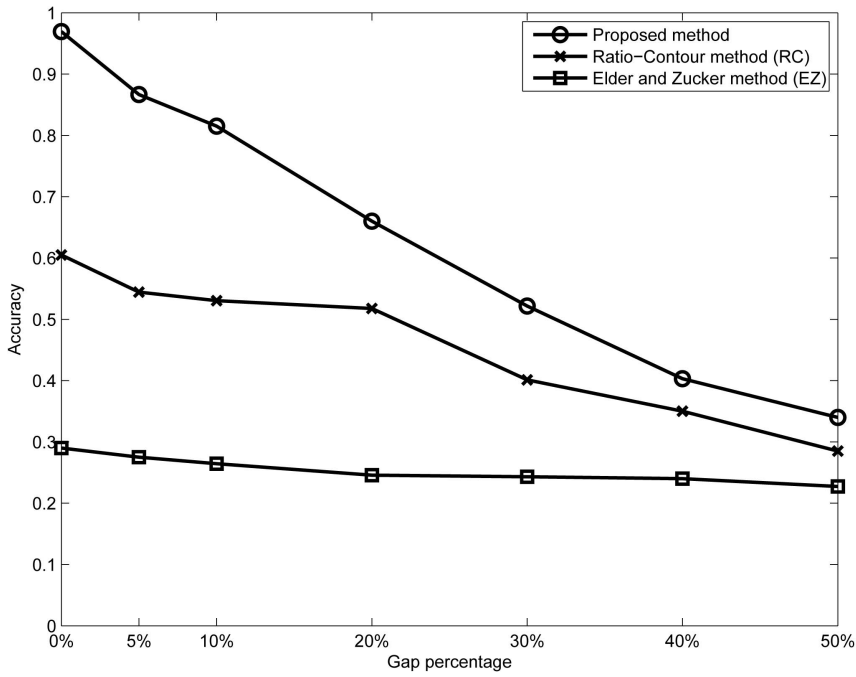

(a)

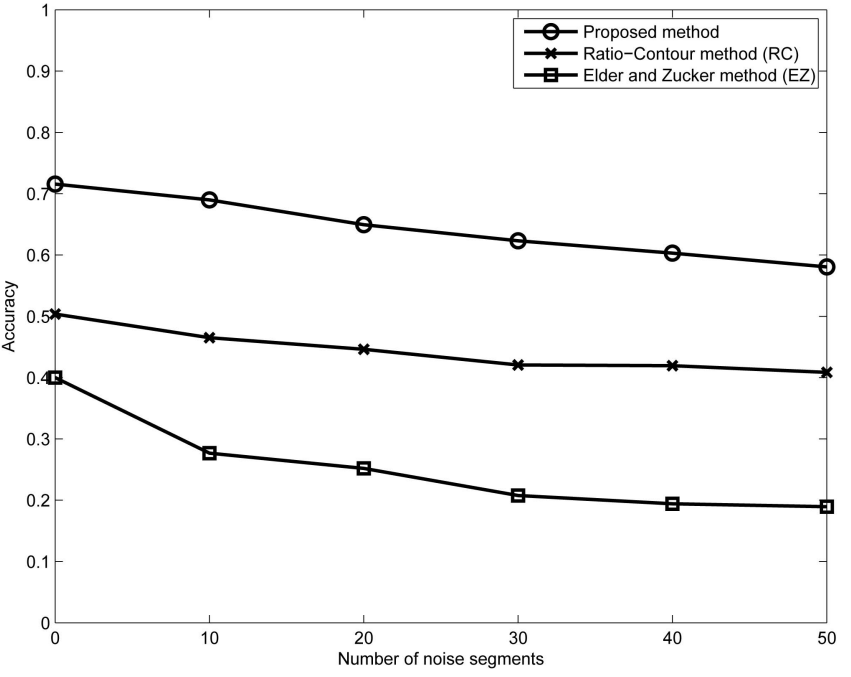

(b)

Fig. 15. The average performance of the proposed method, RC, and EZ on all 2,100 synthetic data samples in terms of (a) the gap percentage along the synthetic boundary pairs and (b) the number of noise segments.

30 percent of the subdivided line segments along the boundary pair shown in Fig. 13a to construct the synthetic data sample in Fig. 14b. Based on such a synthetic data sample, we can directly apply the proposed grouping method to detect an optimal boundary, as shown in Fig. 14c.

For each of the five boundary pairs shown in Fig. 13, we sample them in seven different ways so that the resulting gaps along the boundaries account for 0 percent, 5 percent, 10 percent, 20 percent, 30 percent, 40 percent, and 50 percent of the boundary length, respectively. The number of noise segments are also chosen to be $0,10,20,30,40$, and 50 in different settings. By doing all possible combination of the sampled boundary pairs and the noise segments, we have a total number of $5 \times 6 \times 7=210$ different settings. Under each setting, we further randomly generate the expected number of noise segments and the expected gap percentage along the underlying boundary pair 10 times, which finally results in $210 \times 10=2,100$ synthetic data samples. In the experiments, we apply the proposed grouping method to all these 2,100 synthetic data samples. For comparison, we also apply two previous edge-grouping methods: the RC [37] and the Elder and Zucker method (EZ) [9] to the same 2, 100 synthetic data. The implementation of both $\mathrm{RC}$ and $\mathrm{EZ}$ are the same as in [37]. Note that both RC and EZ consider only boundary closure, proximity, and continuity (smoothness) but not boundary symmetry and enclosed region area, in grouping. Sample grouping results by RC and EZ are shown in Figs. 14d and $14 \mathrm{e}$, respectively.

As in [37], on each data sample, we define the grouping performance using a region coincidence measure $\frac{\left|R \cap R^{\prime}\right|}{\left|R \cup R^{\prime}\right|}$, where $R$ and $R^{\prime}$ are the regions enclosed by the desired ground-truth boundary and the detected boundary, respectively, and $|R|$ indicates the area of region $R$. The larger this measure, the better the coincidence between the ground-truth boundary and the detected boundary. For example, the grouping results shown in Figs. 14c, 14d, and 14e have a performance of 0.98, 0.68 , and 0.19 , respectively. Figs. $15 \mathrm{a}$ and $15 \mathrm{~b}$ show the average performance of the proposed method, $\mathrm{RC}$, and $\mathrm{EZ}$ on all 2,100 data samples, in terms of the gap percentage along the boundary pairs and the number of noise segments, respectively. Note that, for each setting of the gap percentage along the boundary pair in Fig. 15a, the average is taken over 300 data samples since we have five boundary pairs, six levels of the number of noise segments, and 10 rounds of noisesegment and boundary-gap generation. Similarly, for each setting of the number of noise segments in Fig. 15b, the average is taken over 350 data samples since we have five boundary pairs, seven levels of the gap percentage in sampling the boundary pair, and 10 rounds of noise-segment and 
(a)

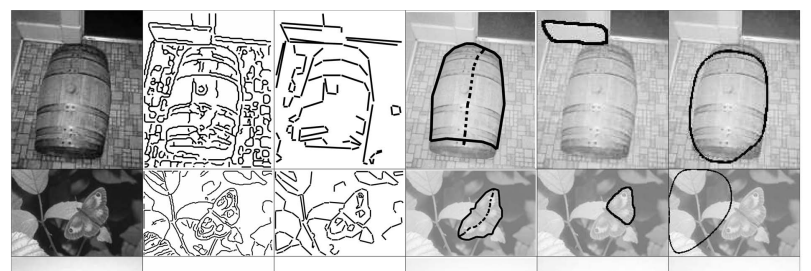

(c)

(d)

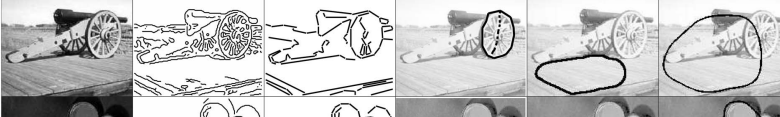

(e)

(f)

(g)

(h)

(i)

(j)

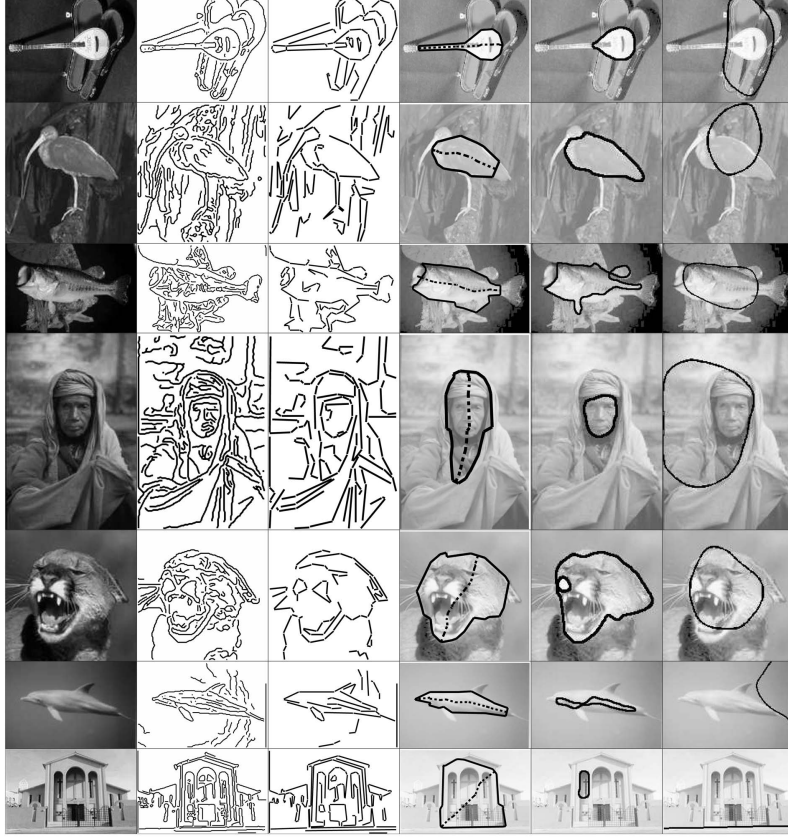

Fig. 16. Grouping results on 10 real images. Column 1: input real images. Column 2: Canny edge detection results. Column 3: detected segments after line fitting. Column 4: optimal boundaries obtained by the proposed grouping method. Detected boundary axes are shown by dashed curves. Column 5: optimal boundaries obtained by RC and Column 6: optimal boundaries obtained by EZ.

boundary-gap generation. From Fig. 15, we can see that, when the desired boundaries are symmetric, the proposed grouping method performs better than RC and EZ, where boundary symmetry is not considered.

\subsection{Experiments on Real Images}

We also test the proposed grouping method on real images and compare it to RC and EZ. The test real images are selected from the Corel image database and Google image search. On real images, we construct the detected segments by edge detection and line fitting. For edge detection, we use the Canny detector provided with Matlab's Image Processing Toolbox (R2006a), leaving its parameters at their default values. For line fitting, we used the Matlab function developed by Kovesi [17] by setting the minimum length for an edge to be considered to 30 pixels and the maximum deviation between an edge and its fitting line to 2 pixels. Sample edge detection and line-fitting results for 20 real images are shown in the second and third columns of Figs. 16 and 17, respectively. Based on these detected segments, all the other settings for the proposed grouping method are the same as the ones used in the above synthetic-data experiments.

The fourth, fifth, and sixth columns in Figs. 16 and 17 show the optimal boundaries obtained by the proposed grouping (a)

(b)

(c)

(d)

(e)
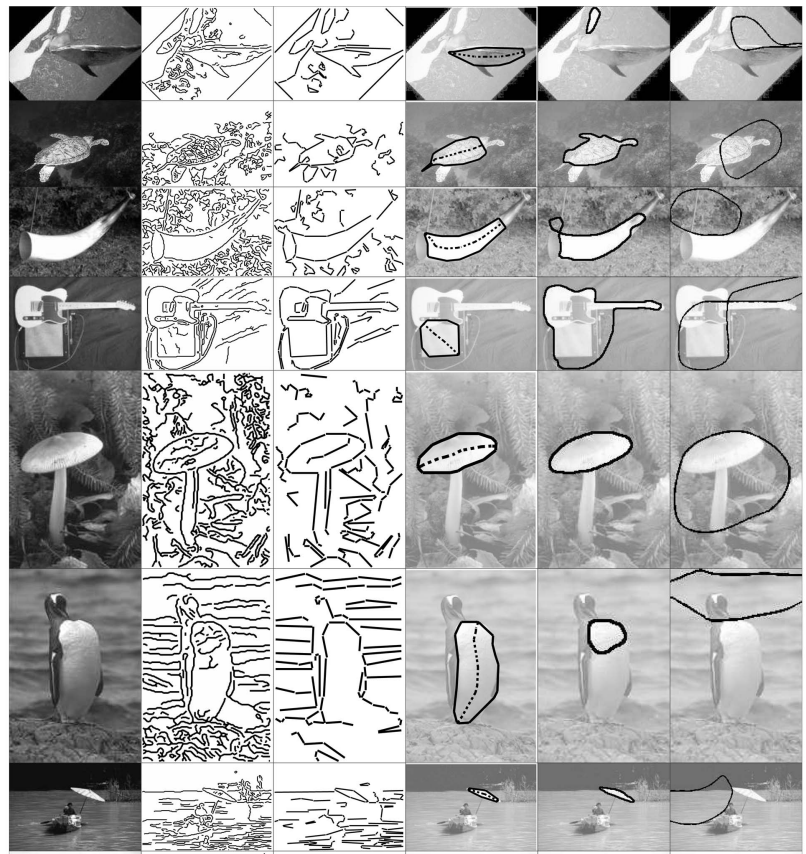

(h)

(i)
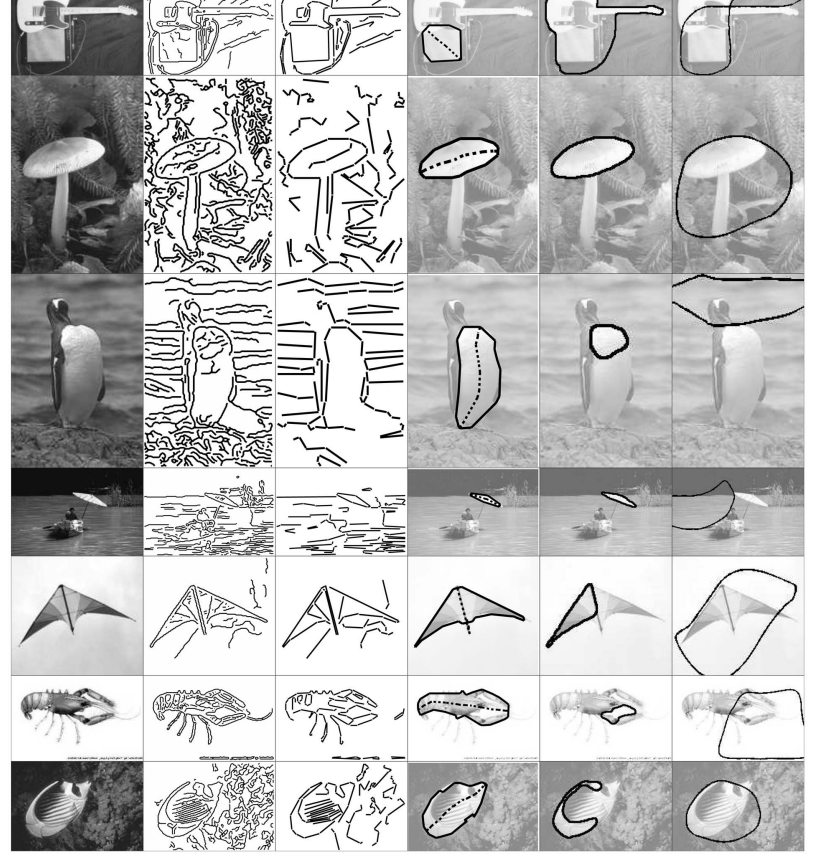

Fig. 17. Grouping results on 10 more real images. The columns depict the same information, as in Fig. 16.

method, RC and EZ, respectively. In Figs. 16a, 16b, 16c, 16d, $16 \mathrm{f}, 16 \mathrm{i}, 16 \mathrm{j}, 17 \mathrm{a}, 17 \mathrm{c}, 17 \mathrm{~d}, 17 \mathrm{f}, 17 \mathrm{~h}, 17 \mathrm{i}$, and $17 \mathrm{j}$, we can see that by considering the symmetry cue the proposed method can detect boundaries with good symmetry. These experiments on real images also show that when the whole structure is not symmetric, the proposed method may only detect a symmetric component of the structure, as shown in Figs. 16c, 16e, $17 \mathrm{a}, 17 \mathrm{~b}, 17 \mathrm{e}$, and $17 \mathrm{f}$. In such cases, the edge-grouping methods without considering symmetry may detect the structural boundary more completely, as shown in Fig. 17b. On several images, such as the ones shown in Figs. 16a, 16e, $17 \mathrm{e}$, and $17 \mathrm{~g}$, RC or EZ produce similar symmetric boundaries as the proposed method does. This indicates that the symmetric boundaries found in these several images by the proposed method also minimize the RC or EZ grouping costs, which consider closure, proximity, and continuity, but not symmetry.

\subsection{Detecting Multiple Boundaries}

In the previous sections, the proposed grouping method is developed and tested to detect the single optimal boundary that minimizes the grouping cost. In practice, most real images contain multiple structures of interest, and there is a strong motivation to extend the proposed grouping method to detect multiple symmetric boundaries from the same image. In addition, due to noise, texture, and other undesirable structures in real images, the optimal boundary that minimizes the grouping cost may not be the desired one. A more reasonable way is to detect a small number of optimal 


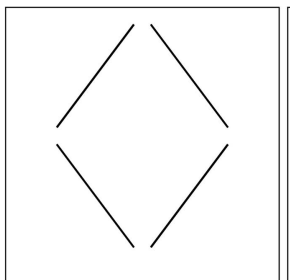

(a)

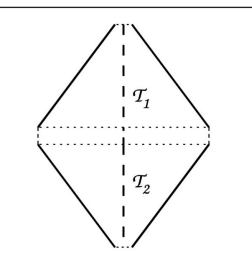

(b)

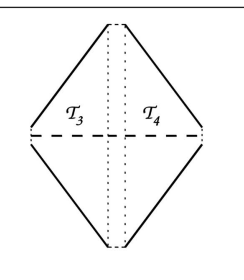

(c)
Fig. 18. An illustration of detecting the same boundary when repeating the proposed grouping method. (a) A set of detected segments. (b) A symmetric boundary consisting of two trapezoids $\mathcal{T}_{1}$ and $\mathcal{T}_{2}$. (c) The same symmetric boundary consisting of two different trapezoids $\mathcal{T}_{3}$ and $\mathcal{T}_{4}$.

boundaries with small grouping cost and expect that the desired ones are among them.

We extend the grouping method to detect multiple optimal boundaries by repeating the proposed grouping method on the same image: after detecting the first optimal boundary, we remove all the trapezoids along the detected boundary and then repeat the same grouping method on the remaining trapezoids to detect the second optimal boundary. This process can be further repeated to detect multiple symmetric boundaries. The implementation of this strategy is simple: each trapezoid corresponds to two edges, and we only need to delete the edges present in the detected optimal cycle (and its mirror cycle) and rerun the minimum-ratio alternate cycle algorithm on the remaining graph to detect the next optimal boundary.

One problem of this multiple-boundary detection is that the same boundary may be detected in different rounds when repeating the proposed grouping algorithm. An example is shown in Fig. 18: the first round of grouping may produce a boundary consisting of the trapezoids $\mathcal{T}_{1}$ and $\mathcal{T}_{2}$. After removing $\mathcal{T}_{1}$ and $\mathcal{T}_{2}$, the second round of grouping may produce a boundary consisting of the trapezoids $\mathcal{T}_{3}$ and $\mathcal{T}_{4}$. We can see that the boundaries produced in these two rounds are in fact the same boundary. However, this mainly happens for a boundary with multiple different symmetry axes, as shown in Figs. 18b and 18c. In practice, this is not a serious problem since we can easily check the detected multiple boundaries and for the boundaries that are detected more than once, we only keep one of them and discard the redundant ones.

We conducted experiments on real images by detecting the first three optimal boundaries, as shown in columns four, five, and six in Fig. 19. We can see that the optimal

(a)

(b)
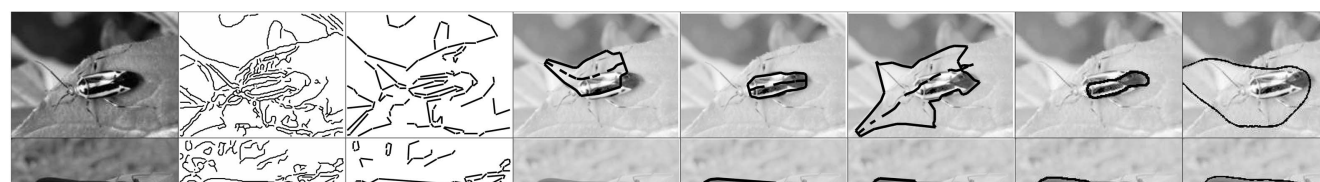

(c)

(d)
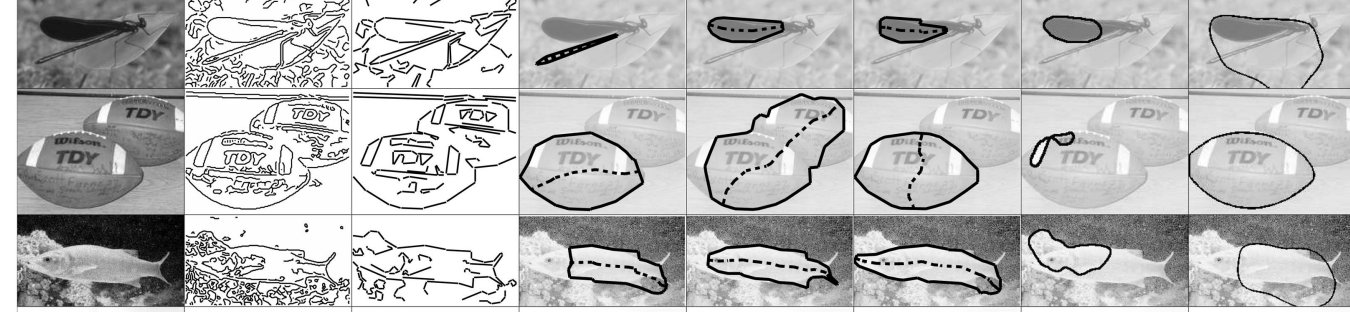

(e)
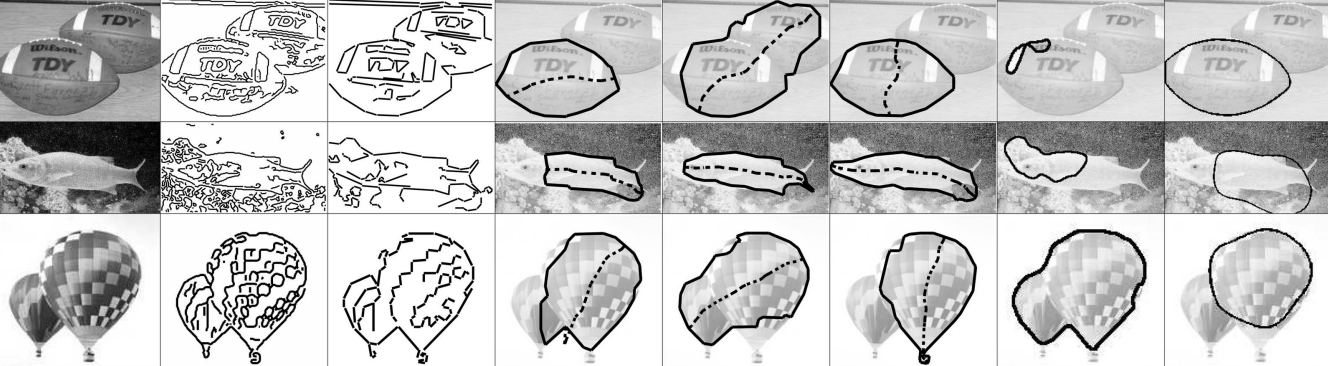

(f)
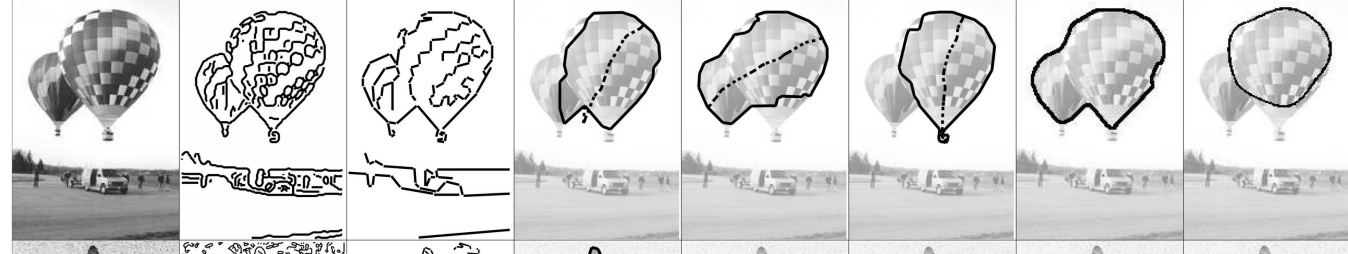

(g)

(h)
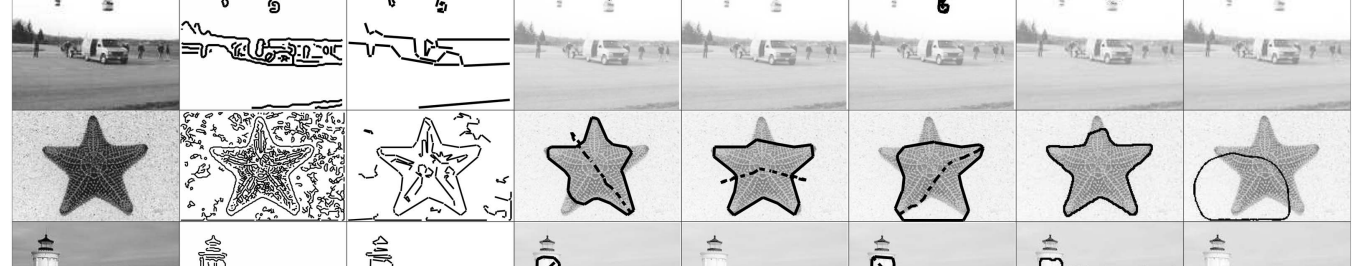

(i)
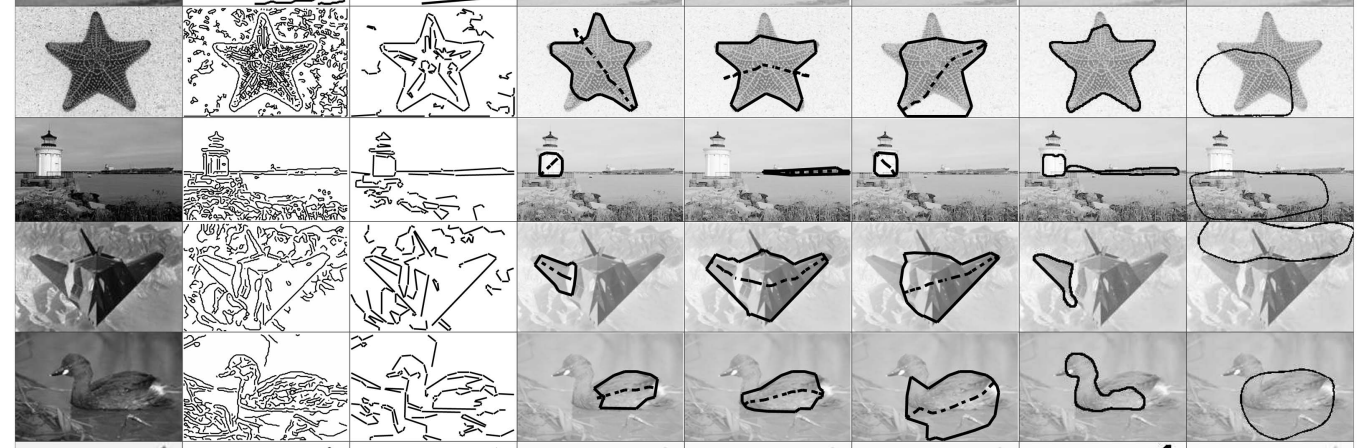

(j)
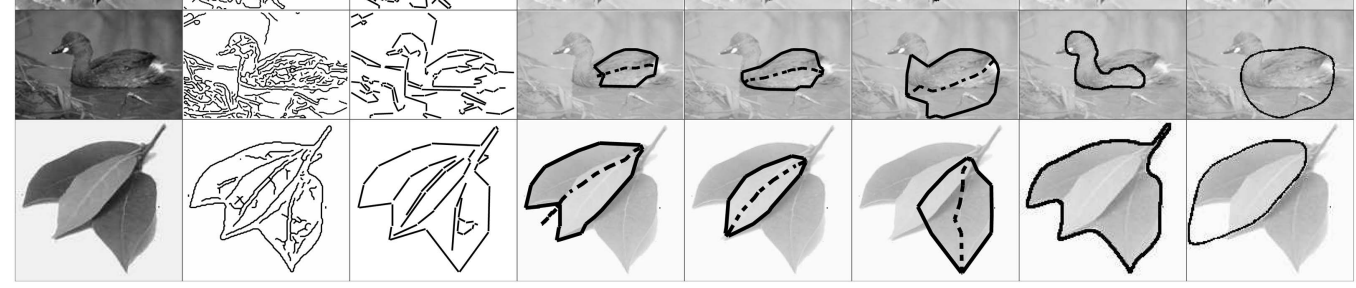

Fig. 19. Experimental results of detecting multiple optimal boundaries from 10 real images. Column 1: input real images. Column 2: Canny edge detection results. Column 3: detected segments after line fitting. Column 4, 5, and 6: the first three optimal boundaries by repeating the proposed grouping method. Column 7: optimal boundaries obtained by RC. Column 8: optimal boundaries obtained by EZ. 
(a)
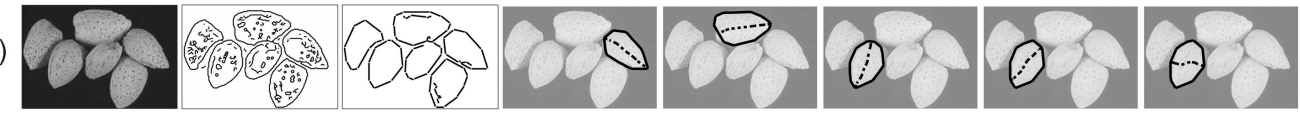

(b)
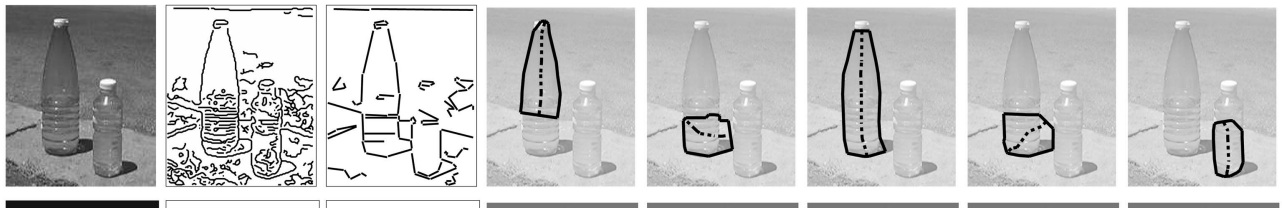

(c)
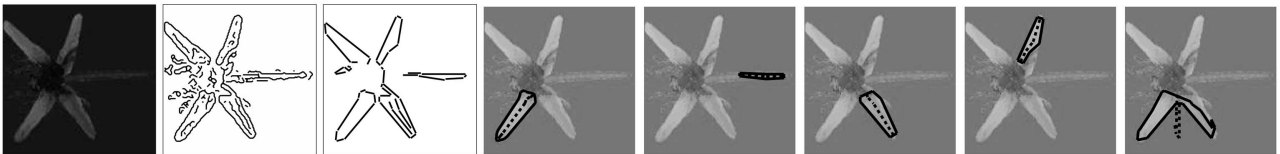

Fig. 20. An illustration of detecting different boundaries or the same boundary in different rounds when repeating the proposed grouping method. From left to right, Column 1: input real images. Column 2: Canny edge detection results. Column 3: detected segments after line fitting. Columns 4, $5,6,7$, and 8 : the first five optimal boundaries by repeating the proposed grouping method.

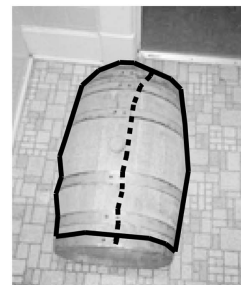

(a)

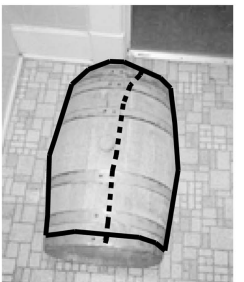

(b)

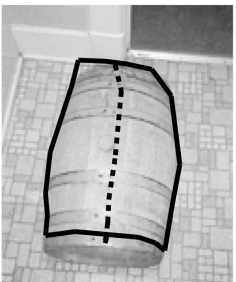

(c)

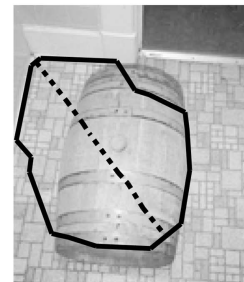

(d)

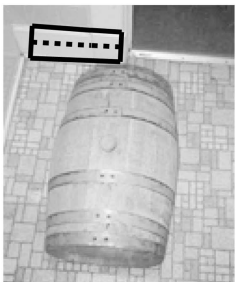

(e)

Fig. 21. An illustration of the optimal boundaries detected by the proposed method using different values of $\lambda$. (a) $\lambda=1$ or 5 , (b) $\lambda=10$ or 25 , (c) $\lambda=50$ or 75 , (d) $\lambda=100$, and (e) $\lambda=1,000$. The input image and the detected segments are the same as the ones shown in Fig. 16a.

boundaries detected in the second or third rounds may be more desirable than the one detected in the first round. In Fig. 20, we further show several examples where the proposed grouping method may detect different symmetric boundaries in different rounds. It also shows an example where the proposed grouping method may detect the same symmetric boundary in different rounds, as shown in columns 6, 7, and 8 in Fig. 20a.

Note that we do not remove all the detected/gap-filling segments along the detected boundary and then repeat the same grouping method on the remaining line segments to detect multiple boundaries. The reason is that removing one segment may correspond to removing many trapezoids, given that the same segment may be used to construct different trapezoids, and this may introduce two problems: 1) for an image with two neighboring structures that share a portion of the their boundaries, the detection of one may prevent the detection of the other, since the segments corresponding to the shared boundary may be removed after the detection of the first boundary and 2) if the boundaries detected in the previous rounds are not the desired ones but include some segments along the desired boundaries, the removal of such segments may prevent the detection of the desired symmetric boundaries in the later rounds.

\subsection{The Selection of $\lambda$ and Other Thresholds}

The proposed grouping cost function (1) has a free parameter $\lambda$, which needs to be selected by the user. This parameter balances the boundary proximity and the boundary symmetry in grouping. A larger $\lambda$ may lead to more symmetric boundaries with poorer proximity, and a smaller $\lambda$ may lead to less symmetric boundaries with better proximity. In most of our experiments, we found that the same or similar grouping results are obtained when $\lambda$ takes a value in a certain range. Fig. 21 shows an example of the proposed grouping with different values of $\lambda$. For this image, when $\lambda$ takes a value in the range $[1.0,50.0]$, the detected boundaries are very similar and well aligned with the barrel present in this image. In general, the selection of $\lambda$ is related to the image size, since the proximity term $\left|\mathcal{B}_{D}\right|$ is related to image size and the symmetric term $\rho(\operatorname{axis}(\mathcal{B}))$ is not. As mentioned earlier, we scale the images to be no larger than $250 \times 250$ while maintaining their aspect ratio in all our experiments on real images. We empirically select $\lambda=10$ for all our experiments.

At the beginning of Section 5, we also introduce several thresholds to reduce the constructed graph size. Particularly, we choose $D_{1}=30, D_{2}=0.5$, and $D_{3}=20$ in our experiments. Using these thresholds, we can avoid constructing the gap-filling quadrilaterals and boundary-axis endpoints that are unlikely to be included in the optimal boundary with the minimum grouping cost. Fig. 22 shows the grouping results by varying the value of these thresholds. By choosing larger values for $D_{1}, D_{2}$, and $D_{3}$, we construct more gap-filling quadrilaterals and consider more potential boundary-axis endpoints. However, the grouping results largely keep unchanged when we choose them to be larger than the ones used in our experiments. In fact, by turning off all three thresholds, that is, setting $D_{1}=+\infty$, $D_{2}=1.0$, and $D_{3}=+\infty$, we get the same grouping result, as shown in the first column in Fig. 22. However, when turning off all of them, the constructed graph has 3,384,762 edges, and the proposed grouping takes 678.69 seconds, compared with 87,292 edges and 3.95 seconds when setting $D_{1}=30$, $D_{2}=0.5$, and $D_{3}=20$.

\subsection{Two Special Cases}

In Section 3.2, we proved that the minimum-ratio alternate cycle contains no more than one auxiliary edge. However, it is 
(a)

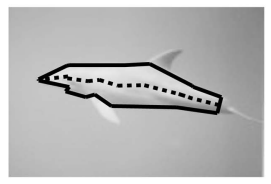

(b)

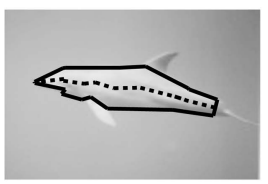

(c)
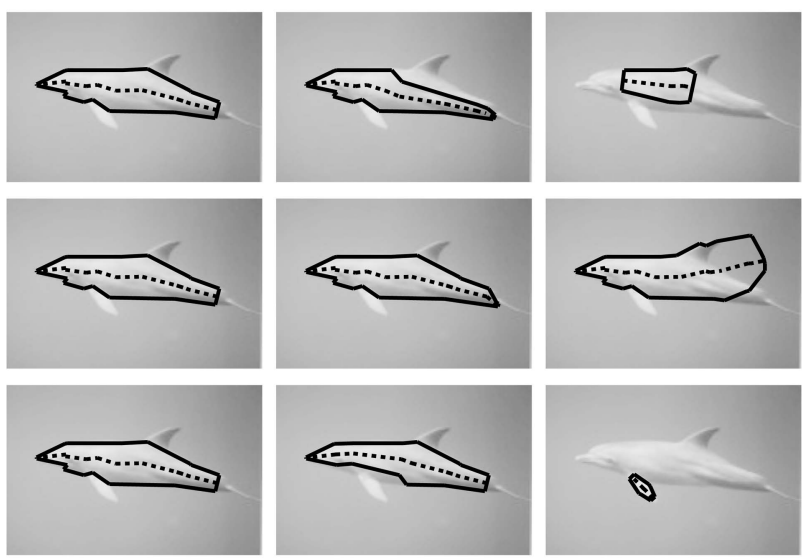

Fig. 22. An illustration of the optimal boundaries detected by the proposed method using different values of $D_{1}, D_{2}$, and $D_{3}$. (a) Fixing $D_{2}=0.5, D_{3}=20$ and varying $D_{1}=25,30,40,50,100,150,200,250$ or $+\infty$ (left), $D_{1}=15$ or 20 (middle) and $D_{1}=5$ or 10 (right). (b) Fixing $D_{1}=30, D_{2}=0.5$ and varying $D_{3}=10,20,30,40,50,100,150$ or $+\infty$ (left), $D_{3}=1,3$ or 5 (middle), and $D_{3}=0$ (right). (c) Fixing $D_{1}=30$, $D_{3}=20$ and varying $D_{2}=0.4,0.5,0.6,0.7,0.8,0.9$ or 1.0 (left), $D_{2}=0.2$ or 0.3 (middle) and $D_{2}=0.1$ (right). The input image and the detected segments are the same as the ones shown in Fig. 16i.

possible that the minimum-ratio alternate cycle does not contain any auxiliary edges. A minimum-ratio alternate cycle without an auxiliary edge may correspond to two special kinds of grouping results that are produced occasionally in practice. The first kind of grouping result consists of two disjoint closed boundaries that are symmetric to each other over an axis in between, as shown in Figs. 23a, 23b, and 23c. Specifically, from the detected segments shown in Fig. 23a, we construct a set of trapezoids, three of which are shown in Fig. 23b. One possible grouping result is to connect these three trapezoids to form two disjoint closed boundaries as shown by the left and right polygons in Fig. 23c, where the middle dashed polygon is the resulting symmetry axis (shown a little misaligned to visualize it better). We can see that the alternate cycle corresponding to this grouping result contains no auxiliary edges, that is, no boundary-axis endpoints can be identified. However, such a grouping result is more likely to have a relatively smaller $\operatorname{area}(\mathcal{B})$ (the total area enclosed by the two resulting disjoint closed boundaries) and a relatively larger $\left|\mathcal{B}_{D}\right|$ (because of the longer total boundary perimeters). Therefore, such a grouping usually has a larger grouping cost and does not happen frequently in the proposed grouping. In Fig. 24, we show an example of such a special grouping result on a real image. This is produced in the second round of repeating the proposed grouping method on this image. In practice, however, we can achieve more such grouping

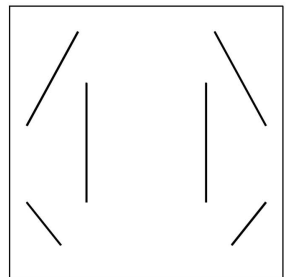

(a)

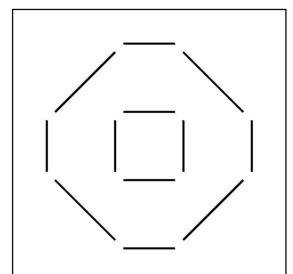

(d)

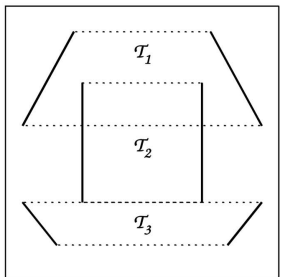

(b)

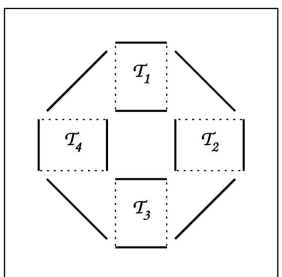

(e)

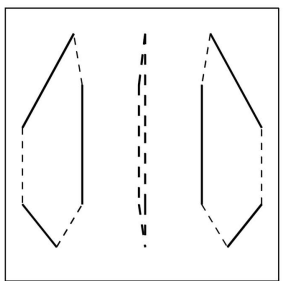

(c)

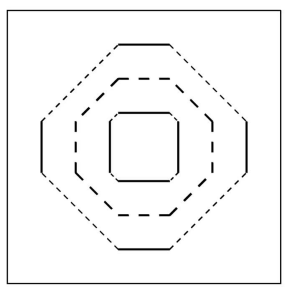

(f)
Fig. 23. An illustration of two special cases in the proposed grouping. Top row: The first special case of detecting a symmetric pair of boundaries. Bottom row: The second special case of detecting two disjoint boundaries that form a ring.

results by not constructing any auxiliary edges in the graph $G=(V, E)$. The application of the same graph algorithm will be forced to detect such symmetric boundary pairs. In practice, this may extend the proposed method to detect the objects in pairs such as eyes, eyeglasses, windows, and so on.

The second kind of special grouping result consists of two disjoint closed boundaries that form a ring, as shown in the second row in Fig. 23. Based on the detected segments shown in Fig. 23d, we can construct trapezoids, as shown in Fig. 23e. One possible grouping result is to connect these four trapezoids to form two disjoint closed boundaries as shown by the innermost and outermost polygons in Fig. 23f, where the polygon between them is the resulting symmetry axis. In this case, the enclosed region area $\operatorname{area}(\mathcal{B})$ is the one bounded by these two disjoint closed boundaries. This special kind of grouping result also occurs very rarely in practice since the enclosed region area is relatively small, and the collinearity of the resulting symmetry axis is usually poor, which results in a large grouping cost. In all our experiments, so far, we did not encounter any such special grouping results.

\section{EXtensions to InCORPORATE Other BOUNDARY AND REGION INFORMATION}

The proposed grouping method can be extended to incorporate other boundary or region information. For example, boundary continuity, or smoothness, is widely
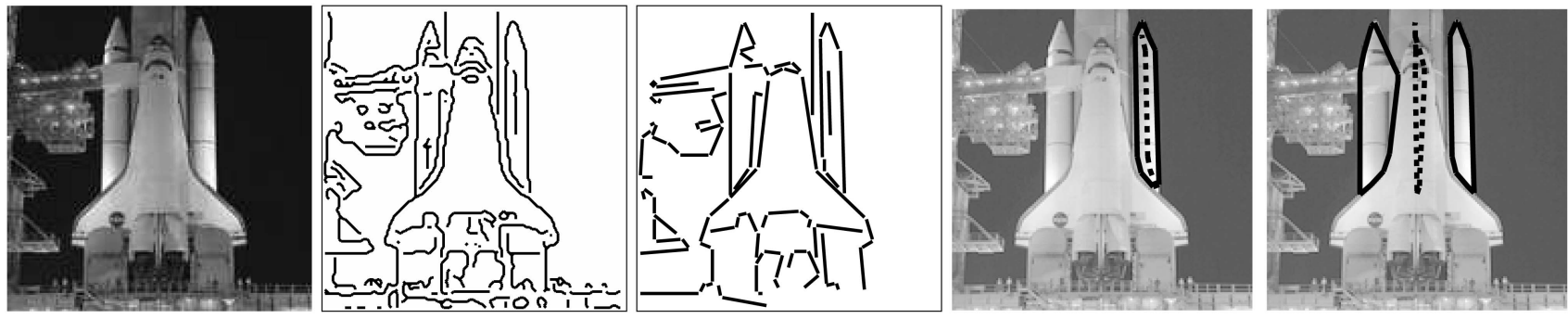

Fig. 24. An example of the special grouping result on a real image. Column 1: input image. Column 2: Canny edge detection result. Column 3: detected segments after line fitting. Columns 4 and 5: the first and second optimal boundaries obtained by repeating the proposed grouping method. 


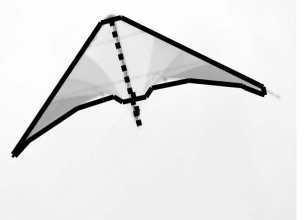

(a)

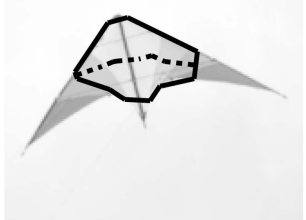

(b)

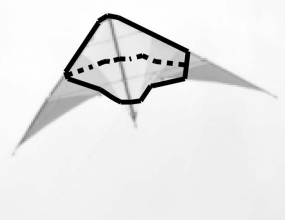

(c)

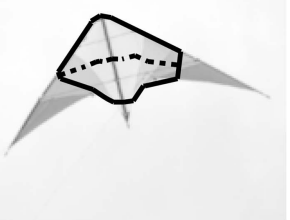

(d)

Fig. 25. An example of the grouping by using the modified grouping cost (3): (a) optimal boundary obtained by the proposed method based on the original grouping cost (1), (b), (c), and (d) optimal boundaries obtained by the proposed method based on the modified grouping cost (3) with $\lambda_{2}=0.5,1$ and 5 , respectively. The input image and the detected segments are the same as the ones shown in Fig. $17 \mathrm{~h}$.

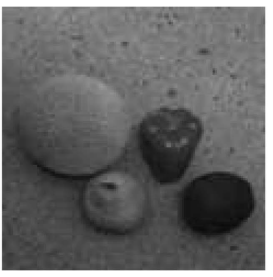

(a)

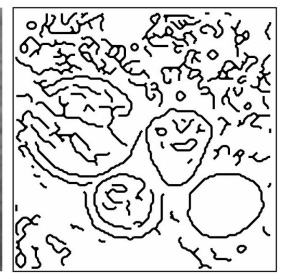

(b)

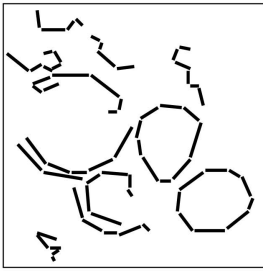

(c)

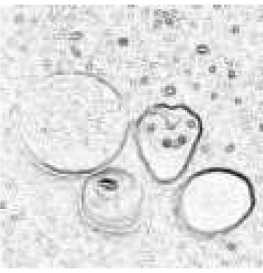

(d)

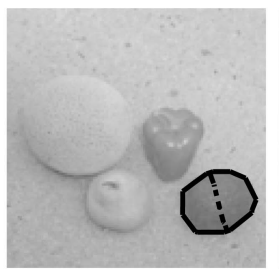

(e)

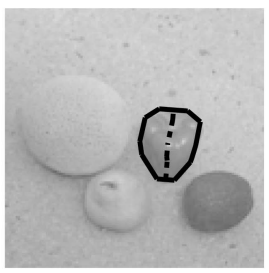

(f)

Fig. 26. An example of grouping by incorporating intensity homogeneity: (a) input real image, (b) Canny edge detection result, (c) detected segments after line fitting, (d) the (normalized) magnitude of the image intensity gradient, where darker pixels indicate larger gradient magnitudes, (e) optimal boundary obtained by the proposed grouping method based on the modified grouping cost (4), and (f) optimal boundary obtained by the proposed method based on the original grouping cost (1).

used in previous edge grouping methods [9], [39], [37], and it can be incorporated in the proposed grouping by adding another term into the numerator of the grouping cost (1) as

$$
\phi(\mathcal{B})=\frac{\left|\mathcal{B}_{D}\right|+\lambda \cdot \rho(\operatorname{axis}(\mathcal{B}))+\lambda_{2} \cdot \int_{\mathcal{B}} \kappa^{2}(t) d t}{\operatorname{area}(\mathcal{B})},
$$

where $\kappa(t)$ is the curvature along the boundary $\mathcal{B}$ and $\lambda_{2}$ is a weighting factor for this continuity term. Since all the considered boundaries are polygons in this paper, cubic spline interpolations are used to estimate the boundary curvature [34].

By making a small change on the first edge weight function, the proposed graph model and algorithm can still be used to find the optimal boundary that minimizes new grouping cost [34]. However, we found that although the boundary continuity is important in previous edge grouping methods, it is not critical in the proposed grouping for symmetric boundaries. The reasons are twofold: 1) with a normalization over the enclosed region area in the grouping cost, the proposed method has a preference to produce boundaries that enclose a large round area. This preference implicitly reflects some level of continuity and 2) as many symmetric boundaries are not smooth everywhere, an explicit inclusion of a curvature term in the grouping cost (3) may affect the detection of such symmetric boundaries. Fig. 25 shows an example where the inclusion of the curvature-based continuity term prevents the detection of a symmetric boundary with high-curvature points.

Other region information can also be incorporated by modifying the denominator of the grouping cost (1). For example, we can extend the proposed method to detect a boundary that encloses a region with good intensity homogeneity by modifying the grouping cost to

$$
\phi(\mathcal{B})=\frac{\left|\mathcal{B}_{D}\right|+\lambda \cdot \rho(\operatorname{axis}(\mathcal{B}))}{\iint_{R(\mathcal{B})}(1-|\nabla I(x, y)|) d x d y},
$$

where $R(\mathcal{B})$ is the region enclosed by the boundary $\mathcal{B}$. Normalized to the range $[0.0,1.0],|\nabla I(x, y)|$ is the magnitude of the intensity gradient at pixel $(x, y)$. To find the optimal boundary that minimizes this modified grouping cost, we only need to modify the definition of the second edge weight in the graph construction from the signed enclosed area $\iint_{R(\mathcal{T})} d x d y$ (or $\left.\iint_{R(\mathcal{G})} d x d y\right)$ to the signed value of $\iint_{R(\mathcal{T})}(1-$ $|\nabla I(x, y)|) d x d y$ (or $\iint_{R(\mathcal{G})}(1-|\nabla I(x, y)|) d x d y$ ), where $R(\mathcal{T})$ (or $R(\mathcal{G})$ ) is the region enclosed by the trapezoid $\mathcal{T}$ (or the quadrilateral $\mathcal{G}$ ) corresponding to the considered edge. The same graph modeling and algorithm can then be used to find the optimal boundary. Fig. 26e shows an example of the grouping using the modified grouping cost (4). As a comparison, Fig. 26f show the grouping result on the same image using the grouping cost (1). We can see that, with this extension, the proposed method detects a boundary whose enclosed region has better intensity homogeneity.

\section{Complexity Analysis and Running Time}

As discussed in Section 5, given $n$ detected segments, we may need to construct $O\left(n^{2}\right)$ trapezoids in the worst case. If we construct quadrilaterals to fill the gaps between each pair of trapezoids, we may then have $O\left(n^{4}\right)$ quadrilaterals, which lead to $O\left(n^{4}\right)$ dashed edges and $O\left(n^{4}\right)$ auxiliary edges in the constructed graph. The minimum-ratio alternate cycle algorithm has a time complexity of $O\left(|V|^{\frac{3}{4}}|E|\right)$ [37] in the worst case. The worst-case complexity of the proposed grouping algorithm is then $O\left(n^{5.5}\right)$. This is a very high complexity and an algorithm with such a complexity is not usually useful in practice.

However, two reasons make the proposed grouping method still practically useful: 1) $n$, the number of detected segments, is usually much smaller than the number of pixels 
TABLE 1

Running Time and the Constructed Graph Size for the 20 Images Shown in Figs. 16 and 17

\begin{tabular}{|l||c|r|r|r|r|r|r|r|}
\hline \hline & Size & Lines & Trapezoids & Quads. & $\begin{array}{r}\text { Auxiliary } \\
\text { Edges }\end{array}$ & $\begin{array}{r}\text { Total } \\
\text { Edges }\end{array}$ & $\begin{array}{r}\text { Prop. Method } \\
\text { CPU(sec) }\end{array}$ & $\begin{array}{r}\text { RC } \\
\text { CPU(sec) }\end{array}$ \\
\hline \hline Fig. 16(a) & $124 \times 150$ & 335 & 10400 & 251154 & 343620 & 605174 & 416.55 & 29.77 \\
\hline Fig. 16(b) & $218 \times 145$ & 580 & 21620 & 523218 & 843700 & 1388538 & 1134.85 & 33.08 \\
\hline Fig. 16(c) & $179 \times 150$ & 353 & 11124 & 256668 & 292612 & 560404 & 325.22 & 28.53 \\
\hline Fig. 16(d) & $176 \times 150$ & 309 & 9282 & 229228 & 286524 & 525034 & 448.60 & 13.29 \\
\hline Fig. 16(e) & $150 \times 161$ & 456 & 17256 & 588778 & 721200 & 1327234 & 2033.32 & 37.53 \\
\hline Fig. 16(f) & $220 \times 150$ & 460 & 14270 & 245566 & 435244 & 695080 & 545.48 & 27.01 \\
\hline Fig. 16(g) & $150 \times 225$ & 559 & 25838 & 699136 & 1307344 & 2032318 & 2468.40 & 68.11 \\
\hline Fig. 16(h) & $150 \times 150$ & 290 & 7672 & 132560 & 135720 & 275952 & 73.85 & 16.85 \\
\hline Fig. 16(i) & $200 \times 133$ & 161 & 3638 & 48010 & 35644 & 87292 & 3.95 & 14.04 \\
\hline Fig. 16(j) & $231 \times 150$ & 512 & 25912 & 899050 & 1547040 & 2472002 & 2192.04 & 57.44 \\
\hline Fig. 17(a) & $210 \times 150$ & 314 & 10210 & 200366 & 205440 & 416016 & 100.97 & 16.82 \\
\hline Fig. 17(b) & $230 \times 150$ & 422 & 11574 & 223262 & 346944 & 581780 & 81.64 & 24.00 \\
\hline Fig. 17(c) & $200 \times 136$ & 538 & 16964 & 321902 & 509040 & 847906 & 698.81 & 29.64 \\
\hline Fig. 17(d) & $212 \times 150$ & 397 & 14102 & 362208 & 459840 & 836150 & 863.27 & 25.62 \\
\hline Fig. 17(e) & $150 \times 225$ & 547 & 19718 & 340776 & 505012 & 865506 & 787.04 & 54.69 \\
\hline Fig. 17(f) & $123 \times 181$ & 334 & 10274 & 166230 & 200344 & 376848 & 246.79 & 30.81 \\
\hline Fig. 17(g) & $225 \times 150$ & 404 & 14324 & 416190 & 424120 & 854634 & 1740.18 & 53.79 \\
\hline Fig. 17(h) & $166 \times 150$ & 143 & 3228 & 58618 & 62304 & 124150 & 6.47 & 12.45 \\
\hline Fig. 17(i) & $231 \times 150$ & 412 & 12710 & 307544 & 448404 & 768658 & 525.75 & 20.04 \\
\hline Fig. 17(j) & $184 \times 125$ & 390 & 13066 & 472338 & 619384 & 1104788 & 1433.91 & 18.56 \\
\hline \hline
\end{tabular}

in an image and 2) we developed several special strategies to substantially reduce the number of edges in the constructed graph (see Section 5). Although it may be difficult to analytically derive a tighter estimate of the algorithm complexity, we implement the proposed grouping method using $\mathrm{C}++$ and check its runtime in processing a set of real images. Table 1 shows the runtime on the 20 images shown in Figs. 16 and 17 and the size of the graph constructed for them. Our experiments were run on Linux computers equipped with a $3.4 \mathrm{GHz}$ Xeon processor and 4 Gbytes of RAM.

\section{Conclusions}

In this paper, we developed a new grouping method for detecting closed boundaries that show good bilateral symmetry. Particularly, the proposed method can detect both boundaries and their symmetry axes. This is achieved by 1) defining a new grouping cost that combines the different boundary and region information, 2) constructing a new type of trapezoidal grouping tokens by pairing line segments detected from the input image, and 3) constructing a new graph model that can transform the proposed grouping problem into a graph problem of detecting a cycle that minimizes a given ratio-form cost. We show that this graph problem can be addressed by an available graph algorithm in polynomial time. We implemented the proposed grouping method and tested its performance on a set of synthetic data and real images. We also conducted experiments to compare its performance to the performance of two previous edge-grouping methods. These experiments showed that the proposed method performs more favorably when the desired structure has a boundary with good bilateral symmetry.

\section{ACKNOWLEDGMENTS}

This work was funded by NSF-EIA-0312861 and AFOSR FA9550-07-1-0250. Part of the experiments were run on the "NICK" Dell PC cluster provided by the College of Engineering and Computing at the University of South Carolina. Some preliminary results of this paper were published in a conference proceeding [34].

\section{REFERENCES}

[1] R.K. Ahuja, T.L. Magnanti, and J.B. Orlin, Network Flows: Theory, Algorithms, and Applications. Prentice Hall, 1993.

[2] T. Alter and R. Basri, "Extracting Salient Contours from Images: An Analysis of the Saliency Network," Int'l J. Computer Vision, vol. 27, no. 1, pp. 51-69, 1998.

[3] A. Amir and M. Lindenbaum, "A Generic Grouping Algorithm and Its Quantitative Analysis," IEEE Trans. Pattern Analysis and Machine Intelligence, vol. 20, no. 2, pp. 168-185, Feb. 1998.

[4] H. Blum, "Biological Shape and Visual Science," J. Theoretical Biology, vol. 38, pp. 205-287, 1973.

[5] H. Blum and R.N. Nagle, "Shape Description Using Weighted Symmetric Axis Features," Pattern Recognition, vol. 10, pp. 167-180, 1978.

[6] M.J. Brady and H. Asada, "Smoothed Local Symmetries and Their Implementation," Technical Report AIM-757, Massachusetts Inst. of Technology, Feb. 1984.

[7] H. Cornelius and G. Loy, "Detecting Bilateral Symmetry in Perspective," Proc. Fifth Workshop Perceptual Organization in Computer Vision, pp. 191-198, 2006.

[8] J.H. Elder, A. Krupnik, and L.A. Johnston, "Contour Grouping with Prior Models," IEEE Trans. Pattern Analysis and Machine Intelligence, vol. 25, no. 6, pp. 661-674, June 2003.

[9] J.H. Elder and S.W. Zucker, "Computing Contour Closure," Proc. European Conf. Computer Vision, pp. 399-412, 1996.

[10] D. Forsyth and J. Ponce, Computer Vision: A Modern Approach. Prentice Hall, 2003.

[11] A. Gupta, V. Prasad, and L. Davis, "Extracting Regions of Symmetry," Proc. IEEE Int'l Conf. Image Processing, vol. 3, pp. 133-136, 2005. 
[12] G. Guy and G. Medioni, "Inferring Global Perceptual Contours from Local Features," Int'l J. Computer Vision, vol. 20, no. 1, pp. 113-133, 1996

[13] H.J.A.M. Heijmans and A.V. Tuzikov, "Similarity and Symmetry Measures for Convex Shapes Using Minkowski Addition," IEEE Trans. Pattern Analysis and Machine Intelligence, vol. 20, no. 9, pp. 980-993, Sept. 1998.

[14] D. Huttenlocher and P. Wayner, "Finding Convex Edge Groupings in an Image," Int'l J. Computer Vision, vol. 8, no. 1, pp. 7-29, 1992.

[15] D. Jacobs, "Robust and Efficient Detection of Convex Groups," IEEE Trans. Pattern Analysis and Machine Intelligence, vol. 18, no. 1, pp. 23-27, Jan. 1996.

[16] G. Kanizsa, Organization in Vision. Praeger, 1979.

[17] P.D. Kovesi, "Matlab Functions for Computer Vision and Image Analysis," School of Computer Science and Software Eng., Univ. of Western Australia, http://www.csse.uwa.edu.au/ pkresearch/ matlabfns/, 2007

[18] S. Lazebnik, C. Schmid, and J. Ponce, "Semi-Local Affine Parts for Object Recognition," Proc. British Machine Vision Conf., vol. 2, pp. $959-968,2004$

[19] M. Leyton, Symmetry, Causality, Mind. MIT Press, 1992.

[20] T. Liu, D. Geiger, and A.L. Yuille, "Segmenting by Seeking the Symmetry Axis," Proc. Int'l Conf. Pattern Recognition, pp. 994-998, 1998.

[21] D.G. Lowe, Perceptual Organization and Visual Recognition. Kluwer Academic, 1985

[22] G. Loy and J.-O. Eklundh, "Detecting Symmetry and Symmetric Constellations of Features," Proc. European Conf. Computer Vision, pp. 508-521, 2006.

[23] S. Mahamud, L.R. Williams, K.K. Thornber, and K. Xu, "Segmentation of Multiple Salient Closed Contours from Real Images," IEEE Trans. Pattern Analysis and Machine Intelligence, vol. 25, no. 4 pp. 433-444, Apr. 2003.

[24] R. Mohan and R. Nevatia, "Perceptual Organization for Scene Segmentation and Description," IEEE Trans. Pattern Analysis and Machine Intelligence, vol. 14, no. 6, pp. 616-635, June 1992.

[25] R.L. Ogniewicz and O. Kübler, "Hierarchic Voronoi Skeletons," Pattern Recognition, vol. 28, no. 3, pp. 343-359, 1995.

[26] V.S.N. Prasad and B. Yegnanarayana, "Finding Axes of Symmetry from Potential Fields," IEEE Trans. Image Processing, vol. 13, no. 12, pp. 1559-1566, 2004.

[27] E. Saber and A. Tekalp, "Frontal-View Face Detection and Facial Feature Extraction Using Color, Shape and Symmetry Based Cost Functions," Pattern Recognition Letters, vol. 19, no. 8, pp. 669-680, 1998.

[28] S. Sarkar and K. Boyer, "Quantitative Measures of Change Based on Feature Organization: Eigenvalues and Eigenvectors," Proc. IEEE Conf. Computer Vision and Pattern Recognition, pp. 478-483, 1996.

[29] A. Shashua and S. Ullman, "Structural Saliency: The Detection of Globally Salient Structures Using a Locally Connected Network," Proc. IEEE Int'l Conf. Computer Vision, pp. 321-327, 1988.

[30] D. Shen, H. Ip, K. Cheung, and E. Teoh, "Symmetry Detection by Generalized Complex (GC) Moments: A Close-Form Solution," IEEE Trans. Pattern Analysis and Machine Intelligence, vol. 21, no. 5, pp. 466-476, May 1999.

[31] H. Shroff and J. Ben-Arie, "Finding Shape Axes Using Magnetic Fields," IEEE Trans. Image Processing, vol. 8, no. 10, pp. 1388-1394, 1999.

[32] K. Siddiqi, S. Bouix, A. Tannenbaum, and S.W. Zucker, "The Hamilton-Jacobi Skeleton," Proc. IEEE Int'l Conf. Computer Vision, vol. 2, pp. 828-834, 1999.

[33] J.S. Stahl and S. Wang, "Convex Grouping Combining Boundary and Region Information," Proc. IEEE Int'l Conf. Computer Vision, vol. 2, pp. 946-953, 2005.

[34] J.S. Stahl and S. Wang, "Globally Optimal Grouping for Symmetric Boundaries," Proc. IEEE Conf. Computer Vision and Pattern Recognition, vol. 1, pp. 1030-1037, 2006.

[35] D. Terzopolous, A. Witkin, and M. Kass, "Symmetry-Seeking Models and 3D Object Reconstruction," Int'l J. Computer Vision, vol. 1, no. 3, pp. 211-221, 1988.

[36] T. Tuytelaars, A. Turina, and L.V. Gool, "Noncombinatorial Detection of Regular Repetitions Under Perspective Skew," IEEE Trans. Pattern Analysis and Machine Intelligence, vol. 25, no. 4, pp. 418-432, Apr. 2003.
[37] S. Wang, T. Kubota, J. Siskind, and J. Wang, "Salient Closed Boundary Extraction with Ratio Contour," IEEE Trans. Pattern Analysis and Machine Intelligence, vol. 27, no. 4, pp. 546-561, Apr. 2005.

[38] S. Wang, J.S. Stahl, A. Bailey, and M. Dropps, "Global Detection of Salient Convex Boundaries," Int'l J. Computer Vision, vol. 71, no. 3, pp. 337-359, 2007.

[39] L. Williams and K.K. Thornber, "A Comparison Measures for Detecting Natural Shapes in Cluttered Background," Int'l J. Computer Vision, vol. 34, no. 2/3, pp. 81-96, 2000.

[40] A.P. Witkin and J.M. Tenenbaum, "On the Role of Structure in Vision," Human and Machine Vision, J. Beck, B. Hope, and A. Rosenfeld, eds., Academic Press, pp. 481-543, 1983.

[41] H. Zabrodsky, S. Peleg, and D. Avnir, "Symmetry as a Continuous Feature," IEEE Trans. Pattern Analysis and Machine Intelligence, vol. 17, no. 12, pp. 1154-1166, Dec. 1995.

[42] S.C. Zhu and A. Yuille, "FORMS: A Flexible Object Recognition and Modelling System," Proc. IEEE Int'l Conf. Computer Vision, pp. $465-472,1995$.

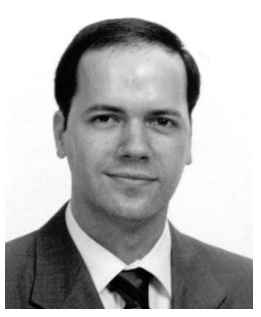

Joachim S. Stahl received the BS degree in computer science from the Augusta State University in 2003 and the ME degree in computer science and engineering from the University of South Carolina in 2005. He is currently a PhD candidate in the Department of Computer Science and Engineering, University of South Carolina. His research interests include computer vision and image processing, including the areas of perceptual organization, segmentation, and image understanding. He is a student member of the IEEE and the IEEE Computer Society.

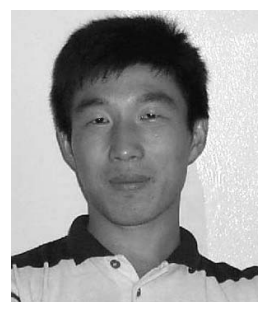

Song Wang received the $\mathrm{PhD}$ degree in electrical and computer engineering from the University of Illinois at Urbana-Champaign in 2002. From 1998 to 2002, he also worked as a research assistant at the Image Formation and Processing Group of the Beckman Institute, University of Illinois at Urbana-Champaign. Since August 2002, he has been an assistant professor in the Department of Computer Science and Engineering at the University of South Carolina. His research interests include computer vision, medical image processing, and machine learning. $\mathrm{He}$ is a member of the IEEE and the IEEE Computer Society.

$\triangleright$ For more information on this or any other computing topic, please visit our Digital Library at www.computer.org/publications/dlib. 